\title{
1 Combining CRISPR/Cas9 and brain imaging: from genes to molecules
}

\section{2 to networks}

3 Sabina Marciano ${ }^{1}$, Tudor Mihai Ionescu ${ }^{1}$, Ran Sing Saw ${ }^{1}$, Rachel Y. Cheong ${ }^{2}$, Deniz Kirik ${ }^{2}$,

4 Andreas Maurer ${ }^{1}$, Bernd Pichler ${ }^{1}$, Kristina Herfert $^{1 \star}$

$11{ }^{1}$ Werner Siemens Imaging Center, Department of Preclinical Imaging and Radiopharmacy,

12 Eberhard Karls University Tuebingen, Germany

$13{ }^{2}$ Brain Repair and Imaging in Neural Systems, Department of Experimental Medical Science, 14 Lund University, Sweden

16 *Corresponding author: Prof. Dr. Kristina Herfert (kristina.herfert@med.uni-tuebingen.de) 


\section{Abstract}

Receptors, transporters and ion channels are important targets for therapy development in neurological diseases including Alzheimer's disease, Parkinson's disease, epilepsy, schizophrenia and major depression. Several receptors and ion channels identified by next generation sequencing may be involved in disease initiation and progression but their mechanistic role in pathogenesis is often poorly understood. Gene editing and in vivo imaging approaches will help to identify the molecular and functional role of these targets and the consequence of their regional dysfunction on whole brain level. Here, we combine CRISPR/Cas9 gene-editing with in vivo positron emission tomography (PET) and functional magnetic resonance imaging (fMRI) to investigate the direct link between genes, molecules, and the brain connectome. The extensive knowledge of the S/c18a2 gene encoding the vesicular monoamine transporter (VMAT2), involved in the storage and release of dopamine, makes it an excellent target for studying the gene networks relationships while structurally preserving neuronal integrity and function. We edited the S/c18a2 in the substantia nigra pars compacta of adult rats and used in vivo molecular imaging besides behavioral, histological, and biochemical assessments to characterize the CRISPR/Cas9-mediated VMAT2 knockdown. Simultaneous PET/fMRI was performed to investigate molecular and functional brain alterations. We found that stage-specific adaptations of brain functional connectivity follow the selective impairment of presynaptic dopamine storage and release. Our study reveals that recruiting different brain networks is an early response to the dopaminergic dysfunction preceding neuronal cell loss. Our combinatorial approach is a novel tool to investigate the impact of specific genes on brain molecular and functional dynamics which will help to develop tailored therapies for normalizing brain function. The method can easily be transferred to higher-order species allowing for a direct comparison of the molecular imaging findings. 
bioRxiv preprint doi: https://doi.org/10.1101/2021.09.10.459766; this version posted October 11, 2021. The copyright holder for this preprint (which was not certified by peer review) is the author/funder, who has granted bioRxiv a license to display the preprint in perpetuity. It is made available under aCC-BY-NC-ND 4.0 International license.

46

47 Keywords

48 CRISPR/Cas9, Functional Connectivity, Dopamine, VMAT2, D2 receptor, $\left[{ }^{11}\right.$ C $]$ raclopride, 49 PET/fMRI.

50 


\section{Introduction}

52 The brain is a network of spatially distributed but functionally and structurally interconnected

53 regions that exhibit correlated activity over time. They communicate with each other via highly

54 specialized neuronal connections and are organized in neuronal circuits and networks.

55 Understanding how functional connections between regions are arranged in the healthy and

56 diseased brain is therefore of great interest.

57 Resting-state functional magnetic resonance imaging ( $r s-f M R I)$ has enabled neuroscientists to

58 delineate the level of functional communication between anatomically separated regions [1]. Rs-

59 fMRI measures the resting-state functional connectivity (rs-FC) at high spatial and temporal

60 resolutions based on spontaneous fluctuations of the blood oxygen level-dependent (BOLD)

61 signal at rest, which indirectly detects neuronal activity via hemodynamic coupling [2]. Using rs-

$62 \mathrm{fMRI}$ several brain resting-state networks in humans and rodents have been identified, such as

63 the default mode and sensorimotor networks (DMN, SMN) [3-9]. Alterations of these networks are

64 linked to neurological diseases [10, 11], and may serve as early therapeutic and diagnostic

65 biomarkers. However, the molecular signatures related to the functional alterations in disease

66 remain largely unknown.

67 Positron emission tomography (PET) provides a non-invasive tool to indirectly measure molecular

68 changes in the brain with high specificity and sensitivity. One well-characterized example is the

69 radioligand $\left[{ }^{11} \mathrm{C}\right]$ raclopride, a widely used D2/D3 receptor antagonist enabling the non-invasive

70 determination of dopamine release and availability [12-14].

71 PET in combination with BOLD-fMRI has the great potential to investigate the molecular

72 substrate of brain functional connectivity (FC), enabling the direct spatial and temporal correlation

73 of both measurements [15-21]. In this context, we have recently shown that rs-FC is modulated

74 by intrinsic serotonin transporter and D2/3 receptor occupancy in rats [22]. 
75 Insights into functional brain circuits and their relationships to individual phenotypes can be

76 gained by genetic manipulations of neuronal subtypes [23]. Genome-engineering methodologies

77 based on clustered regularly interspaced short palindromic repeats (CRISPR)/associated RNA-

78 guided endonuclease (Cas9) represent a promising approach to unveil the influence of genes on

79 brain circuits. CRISPR/Cas9 has enabled researchers to interrogate the mammalian DNA in a

80 precise yet simple manner $[24,25]$ in several species [26-31], by editing single or multiple

81 genomic loci in vitro and in vivo [25, 32, 33]. However, one great hurdle is the brain delivery,

82 which must comply with effective nuclear access, while minimizing immunogenic reactions and

83 off-target editing [34]. Despite these limitations, the potential of CRISPR/Cas9 is continuously

84 expanding with novel nuclease variants being exploited [35-37]. Derived from Staphylococcus

85 aureus, SaCas9 overcomes the packaging constraints of adeno-associated viral vectors (AAVs),

86 allowing efficient CRISPR/Cas9 brain transfer [38-42].

Here, we use an AAV-based CRISPR/SaCas9 gene-editing approach to knock down the S/c18a2 gene encoding the vesicular monoamine transporter 2 (VMAT2), a key protein involved in the storage and release of dopamine in the brain [43]. The extensive knowledge on Slc18a2 makes it an excellent basis for studying the gene networks relationships. We characterize the VMAT2mediated dopamine signaling using in vivo molecular imaging, behavioral, histological, and biochemical assessments. We investigate the impact of impaired VMAT2-dependent dopamine

93 neurotransmission on the DMN and SMN using a simultaneous $\left[{ }^{11} \mathrm{C}\right]$ raclopride-PET/fMRI 94 protocol. Further, we dig into the dopamine-GABA interplay using $\left[{ }^{11} \mathrm{C}\right]$ flumazenil PET. Our results reveal that CRISPR/SaCas9-induced synaptic dysfunction prompts early network changes, preceding motor and molecular alterations, including a regional increase in postsynaptic dopamine receptor availability. We identify a pattern of asymmetric hyperconnectivity, and internetwork synchronization, spreading from the contralateral thalamus (SMN), and prefrontal 
101 Our findings illustrate the ability of the brain to recruit different brain networks and functionally 102 compensate for the dopaminergic dysfunction prior to neuronal cell loss, postsynaptic changes, 103 and motor impairment.

\section{Results}

In vitro validation of CRISPR/SaCas9-induced VMAT2 knockdown in rat primary cortical neurons

To evaluate the efficiency of the AAV-based CRISPR/SaCas9 VMAT2 knockdown in rat primary neurons, we designed AAV-SaCas9 and AAV-sgRNA targeting the first exon of the bacterial lacZ gene (control) or the second exon of the Slc18a2 gene (Fig. 1a) (sgRNAs sequences are reported in Table 1). Seven days post-transduction, the protein expression level and mutation rate of the harvested genomic DNA were inspected by immunofluorescence and surveyor assay (Fig. 1b). Immunofluorescence indicated a clear reduction of VMAT2 protein expression in neurons transduced with AAV-SaCas9 and AAV-sgRNA-Slc18a2 (Fig. 1c). We observed 20\% editing for the digested DNA from neurons transduced with vectors for SaCas9 and sgRNASlc18a2 (Supplementary Fig.1). terminal loss or neuroinflammation in the adult rat brain

To test the in vivo efficiency of the CRISPR/SaCas9 gene-editing, we expressed SaCas9 and sgRNA targeting Slc18a2 to knock down the VMAT2, or targeting lacZ as control, by AAVmediated gene transfer into the right substantia nigra pars compacta (SNc). DPBS was injected 123 into the left SNc. $\left[{ }^{11} \mathrm{C}\right]$ Dihydrotetrabenazine (DTBZ) PET imaging was performed $8-10$ weeks post-injection to quantify VMAT2 expression in the striatum (Fig. 2a). 
$125\left[{ }^{11}\right.$ C]DTBZ binding was decreased by $30 \%(0-62 \%)$ in the right striatum of rats where the VMAT2 was knocked down in comparison to the contralateral striatum. No changes of $\left[{ }^{11} \mathrm{C}\right] \mathrm{DTBZ}$ binding were observed in the contralateral striatum, as $\left[{ }^{11} \mathrm{C}\right] \mathrm{DTBZ}$ binding did not differ between the left striatum of rats injected with sgRNA targeting lacZ and rats injected with sgRNA targeting Slc18a2 (Fig. 2b,c).

We further evaluated changes of dopamine availability in the striatum using $\left[{ }^{11} \mathrm{C}\right]$ raclopride, which competes with dopamine for the same binding site at the D2 receptor (D2R) [13]. After 12 14 weeks following CRISPR/SaCas9-induced VMAT2 knockdown in nigrostriatal neurons, we observed $17 \%$ increased binding of $\left[{ }^{11} \mathrm{C}\right]$ raclopride in the right striatum of VMAT2 knockdown rats and no changes in control rats (Fig. 2b,c), indicating a reduction of synaptic dopamine levels and/or compensatory changes of $\mathrm{D} 2 \mathrm{R}$ expression at postsynaptic medium spiny neurons. A larger VMAT2 knockdown led to lower dopamine levels in the striatum and thus to higher D2R binding (Fig. 2d). To explore the threshold at which the observed postsynaptic changes occur, we calculated the $\left[{ }^{11} \mathrm{C}\right]$ raclopride $/\left[{ }^{11} \mathrm{C}\right] \mathrm{DTBZ}$ binding ratio for the right and left striatum. The ratio remained close to 1 in the DPBS-injected striatum and control rats, indicating no substantial difference between the two hemispheres. In contrast, VMAT2 knockdown rats displayed large

$141\left[{ }^{11} \mathrm{C}\right]$ raclopride binding changes when the level of VMAT2 knockdown was $\sim 20 \%$. From this 142 point, a prominent increase in D2R binding was observed in the right striatum (Fig. 2e). 143 Therefore, this threshold was set to split the rats into mild $(<20 \%)$ and moderate $(\geq 20 \%)$. 144 Notably, $\left[{ }^{11} \mathrm{C}\right]$ raclopride PET imaging was able to discriminate between different degrees of 145 synaptic dysfunction, classified from $\left[{ }^{11} \mathrm{C}\right] \mathrm{DTBZ}$ binding changes (Fig. 2f).

146 We inspected the integrity of dopaminergic nerve terminals and the occurrence of 147 neuroinflammation in the striatum after the CRISPR/SaCas9-induced VMAT2 knockdown. $148\left[{ }^{11} \mathrm{C}\right]$ methylphenidate PET imaging of the dopamine transporter and $\left[{ }^{18} \mathrm{~F}\right] \mathrm{GE}-180$ PET imaging of 149 the translocator protein, which is overexpressed on activated microglia, was performed. 
154 To explore the motor consequences of the CRISPR/SaCas9-induced VMAT2 knockdown, we performed several behavioral tests (Fig. 3a).

We observed a reduction in the locomotor activity of VMAT2 knockdown rats in the open field test

Next, we evaluated the forelimb akinesia using the cylinder test. VMAT2 knockdown rats displayed a preference for the right forepaw, while control rats equivalently used their right and left forepaw (Fig. 3c). Paw use alterations correlated highly with VMAT2 knockdown $\left(\Delta\left[{ }^{11} C\right] D T B Z\right.$ binding), and dopamine availability ( $\Delta\left[{ }^{11} \mathrm{C}\right]$ raclopride binding) (Fig. 3d,e).

To further examine differences in motor function, coordination, and balance, rats underwent the beam walk test. VMAT2 knockdown rats stumbled with higher frequency to the left side, while control rats displayed equal chances to slip in each direction (Fig. 3f). However, no correlations between gait alterations and VMAT2 knockdown ( $\Delta\left[{ }^{11} \mathrm{C}\right] \mathrm{DTBZ}$ binding), or dopamine availability $\left(\Delta\left[{ }^{11} \mathrm{C}\right]\right.$ raclopride binding) were found (Supplementary Fig. 2c,d).

As previous studies suggest that body weight changes reflect striatal dopamine depletion [44], we inspected the impact of the VMAT2 knockdown on the rats' body weight gain. VMAT2 knockdown rats exhibited a $30 \%$ reduction in their gained weight over a period of 14 weeks, compared with controls (Fig. 3g). Body weight gain correlated with changes in VMAT2 expression $\left(\Delta\left[{ }^{11} \mathrm{C}\right] \mathrm{DTBZ}\right.$ binding), and dopamine availability ( $\Delta\left[{ }^{11} \mathrm{C}\right]$ raclopride binding) (Fig. 3h,i).

173 To assess the rotational behavior, we performed the rotameter test with and without apomorphine 174 administration. In the spontaneous rotation test, VMAT2 knockdown rats displayed a higher 175 number of ipsilateral net turns compared with control rats (Fig. 3j). The number of turns did not 
176 correlate with VMAT2 expression changes ( $\Delta\left[{ }^{11} \mathrm{C}\right] \mathrm{DTBZ}$ binding), and changes in dopamine

177 availability ( $\Delta\left[{ }^{11} \mathrm{C}\right]$ raclopride binding) (Supplementary Fig. 2e,f). Apomorphine-induced rotations

178 to the contralateral side were higher in VMAT2 knockdown rats compared with control rats (Fig.

$1793 \mathrm{k}$ ), and correlated with changes in VMAT2 expression ( $\Delta\left[{ }^{11} \mathrm{C}\right] \mathrm{DTBZ}$ binding) and dopamine 180 availability ( $\Delta\left[{ }^{11} \mathrm{C}\right]$ raclopride binding) (Fig. 3l,m).

\section{Ex vivo validation of the CRISPR/SaCas9-induced VMAT2 knockdown}

183 Using immunofluorescence, we confirmed the concomitant expression of SaCas9 and S/c18a2184 targeting sgRNA 19 weeks post-transduction, and a corresponding decrease of VMAT2 185 expression in the SNc of the VMAT2 knockdown group (Fig. 4b).

186 Immunohistochemistry revealed no changes in tyrosine hydroxylase (TH) expression levels in 187 striatum and SN in both groups (Fig. 4c,d), and confirmed the reduction of VMAT2 expression in 188 the right striatum and SN in the knockdown group (Fig. 4e).

Biochemical analysis showed a large reduction of dopamine, paralleled by an increased ratio of metabolites (DOPAC, HVA) to dopamine, in the right striatum of VMAT2 knockdown rats (Fig. $\left.\mathrm{BP}_{\mathrm{ND}}\right)$ and postsynaptic changes $\left(\Delta\left[{ }^{11} \mathrm{C}\right] \mathrm{RAC} \mathrm{BP}_{\mathrm{ND}}\right)$ (Fig. 4h,i). Additionally, serotonin was unchanged in the striata of VMAT2 knockdown and control rats, suggesting dopamine nigrostriatal pathway specificity (Fig. 4j) (Metabolites' and neurotransmitters' striatal levels are reported in Table 3). knockdown

199 As multiple lines of evidence suggest a broader role of dopamine in the dynamic reconfiguration 200 of brain networks $[17,45,46]$, we next investigated the impact of unilateral dopamine depletion 201 on brain rs-FC. A second cohort of rats underwent longitudinal simultaneous $\left[{ }^{11} \mathrm{C}\right]$ raclopride- 
PET/BOLD-fMRI scans at baseline and 8 - 14 weeks after CRISPR/SaCas9-induced VMAT2 knockdown (Fig. 5a). $\left[{ }^{11}\right.$ C]DTBZ PET scans and behavioral analysis confirmed previous findings in the first cohort, that is, an efficient depletion of the VMAT2 (20\% decrease of $\left[{ }^{11}\right.$ C]DTBZ binding) (Supplementary Fig. 3a), paralleled by motor disturbances in the cylinder test (Supplementary Fig. 3e-g). In line with the findings of cohort 1, dopamine availability was decreased (10\% increase in $\left[{ }^{11} \mathrm{C}\right]$ raclopride binding) and correlated to the extent of the VMAT2 knockdown (Supplementary Fig. 3b,c). An increase in D2R binding was observed in the right striatum when the level of VMAT2 knockdown reached 20\% (Supplementary Fig. 3d), enabling a subdivision into mild $(<20 \%)$ and moderate rats $(\geq 20 \%)$.

We next assessed the occurrence of rs-FC changes in DMN and SMN. Our analysis focused on identifying early biomarkers of mild dysfunction and patterns of spreading of synaptic dysfunction. Figure 5b,c illustrates intraregional rs-FC group-level correlation matrices at baseline and after

214 VMAT2 knockdown in mild and moderate rats for the DMN and SMN, respectively. We observed within-network rs-FC changes in rats with moderate VMAT2 knockdown, in both DMN and SMN. Rats of the mild knockdown group revealed rs-FC changes up to $20 \%$, in prefrontal cortical regions of the DMN, and between the left thalamus (Th) and somatosensory cortex (SC) in the

218 SMN. However, these data need to be carefully interpreted as they did not survive a more 219 stringent $P$ value selection $\left({ }^{\star} P<0.01\right)$ (Supplementary Fig.4a,b) $(P$ values are reported in 220 Supplementary Tables 1,3).

221 Rats with moderate VMAT2 knockdown exhibited a 60\% increase in rs-FC within the right medial 222 prefrontal cortex (mPFC) and the right and left hippocampus (Hipp) (Fig. 5b) ( $P$ values are 223 reported in Supplementary Table 2).

224 FC increase between the left thalamus and somatosensory cortex in the SMN doubled to $34 \%$ in 225 rats with moderate VMAT2 knockdown and extended throughout the left and right thalamus and 226 striatum (STR), respectively (Fig. 5c) ( $P$ values are reported in Supplementary Table 4). 
227 Moreover, we inspected rs-FC changes between the DMN and SMN at baseline and after the

228 CRISPR/SaCas9-induced VMAT2 knockdown.

229 Figure 5d illustrates internetwork rs-FC correlation matrices in rats with mild (left panel) and 230 moderate (right panel) VMAT2 knockdown. Brain graphs display the nodes and edges (raw 231 values) that demonstrated internetwork rs-FC changes to baseline (\%). Strikingly, large 232 alterations between DMN and SMN were already observable in the mild VMAT2 knockdown 233 group. Rats presented opposite rs-FC changes between regions of the anterior/posterior DMN 234 and the SMN, compared with baseline. A 30 to $60 \%$ increase in rs-FC was observed between 235 regions of the anterior DMN and the SMN. Specifically, rs-FC increased between the right 236 orbitofrontal cortex (OFC) and striatum bilaterally and the contralateral somatosensory cortex, 237 and between the contralateral orbitofrontal cortex and striatum. Instead, a 20\% decrease in rs-FC 238 was found between regions of the posterior DMN and the SMN. Specifically, rs-FC decreased 239 between the left retrosplenial cortex (RSC) and right somatosensory cortex (Fig. 5d, left panel) $(P$ 240 values are reported in Supplementary Table 5).

241 Rats with a moderate VMAT2 knockdown presented increased rs-FC between regions of the 242 anterior/posterior DMN and the SMN, compared with baseline. Of particular note, internetwork rs243 FC changes were not found between the regions of the posterior DMN and the SMN that showed 244 decreased rs-FC in rats with mild VMAT2 knockdown. Moreover, between-network rs-FC 245 increase extended to other regions. A 60 to $80 \%$ increase in rs-FC was found between the medial 246 prefrontal cortex and the right striatum, and the motor (MC) and somatosensory cortex bilaterally.

247 FC increased by more than $20 \%$ between the hippocampi and contralateral somatosensory 248 cortex. (Fig. 5d, right panel) ( $P$ values are reported in Supplementary Table 6). Notably, between249 network rs-FC changes did not involve the thalamus, which connectivity was however altered 250 within the SMN.

251 Further, we examined how the rs-FC changes to baseline correlated between the mild and 252 moderate groups (Supplementary Fig. 5a). Group level intraregional and internetwork rs-FC 
changes to baseline (\%) correlated linearly between the two groups (Supplementary Fig. 5b-d). Node correlation analysis indicated a linear increase in the magnitude of the rs-FC changes to baseline in the hippocampi (Supplementary Fig. 5e), cingulate cortices (Supplementary Fig. 5f), and contralateral, but not ipsilateral, thalamus (Supplementary Fig. $5 \mathrm{~g}$ ). Our data suggest a similarity in the pattern of the intraregional and internetwork rs-FC changes between mild and moderate VMAT2 knockdown rats and a linear relationship between the magnitude of the rs-FC changes.

To complement the results of the intraregional and internetwork rs-FC, we evaluated changes in regional mean connection distances. Network-wise graph theoretical analysis on node level was paralleled by whole-brain connection-wise analysis to identify the nodes that were significantly altered in rats with mild and moderate VMAT2 knockdown, compared with baseline, for the DMN (Supplementary Fig. 6a,b) and SMN (Supplementary Fig. 6c,d). Briefly, the network organization did not change in rats with mild VMAT2 knockdown, compared with baseline, as changes in the global mean connection distance were not found in DMN (Supplementary Fig. 6a) nor SMN (Supplementary Fig. 6c). Interestingly, in rats with moderate VMAT2 knockdown network organization changes did not influence regions of the DMN (Supplementary Fig. 6b), but occurred 269 in the contralateral striatum and thalamus (Supplementary Fig. 6d) $(P$ values are reported in 270 Supplementary Table 7).

271 Collectively, rs-FC results highlight lateralized effects in the SMN, as opposed to the symmetric 272 recruitment of DMN regions.

275 Besides dopamine, dopaminergic neurons co-release GABA via the VMAT2 [47, 48]. To 276 investigate if GABA neurotransmission is altered following the VMAT2 knockdown, we performed 277 additional $\left[{ }^{11} \mathrm{C}\right]$ flumazenil PET scans $10-12$ weeks after the CRISPR/Cas9-editing, and 

cortex (PaC) (14\%), hippocampus (4\%), and somatosensory cortex (9\%) (Fig. 6a,b). In moderate VMAT2 knockdown rats we observed a decrease of $\left[{ }^{11} \mathrm{C}\right]$ flumazenil binding in the ipsilateral parietal cortex (11\%), and somatosensory cortex (6\%) (Fig. 6a,c). Our data indicate that $\left[{ }^{11} \mathrm{C}\right]$ flumazenil binding was altered regardless of the VMAT2 knockdown extent. This was further evidenced by the lack of correlation between $\left[{ }^{11} \mathrm{C}\right] \mathrm{DTBZ}$ and $\left[{ }^{11} \mathrm{C}\right]$ flumazenil binding changes in the target regions (linear regression data not shown).

\section{Discussion}

Here, we show the potential of combining CRISPR/Cas9 gene-editing with molecular and functional brain imaging to identify early adaptations of brain circuits in response to targeted gene and protein modulations. Using CRISPR/SaCas9, we knocked down the Slc18a2 gene, encoding the VMAT2, which plays a key role in the storage and release of dopamine in response to neuronal activity [43]. The CRISPR-mediated knockdown allowed us to investigate the VMAT2dependent dopamine signaling in the striatum, while structurally preserving neuronal integrity. $\left[{ }^{18} \mathrm{~F}\right] \mathrm{GE}-180$ results suggest that glial activation is not the source of dopaminergic synaptic dysfunction, and exclude the occurrence of inflammatory responses arising from the surgical procedure and the chosen AAV-serotype, which could influence our readout, in line with recent reports [49]. Our data reveal that the targeted gene knockdown in the SNc leads to an expected reduction of dopamine release in the striatum, paralleled by $\left[{ }^{11} \mathrm{C}\right]$ raclopride binding changes. It is conceivable that the observed postsynaptic changes are the result of an increase in binding due to the reduced dopamine concentration in the striatum. Yet, several studies have revised this notion $[50,51]$. In this regard, our results of the drug-evoked rotational behavior are in better agreement with a D2R compensatory upregulation. Indeed, supersensitivity to apomorphine in 
binding sites, but no change in affinity [52-57]. Further, the observed striatal increase in $\left[{ }^{11} \mathrm{C}\right]$ raclopride binding is independent of presynaptic nerve terminal loss and occurs in response to $\mathrm{a} \sim 20 \%$ decrease of $\left[{ }^{11} \mathrm{C}\right] \mathrm{DTBZ}$ binding. This confirms, in line with our $\left[{ }^{11} \mathrm{C}\right] \mathrm{methylphenidate}$ results, that $\left[{ }^{11} \mathrm{C}\right]$ raclopride can be used to delineate postsynaptic changes in the absence of dopamine transporter-mediated compensation, triggered by neuronal loss. Accordingly, increased $\left[{ }^{11} \mathrm{C}\right]$ raclopride binding is observed in the early but not later stages of Parkinson`s disease [58], characterized by severe neuronal cell loss, and dopamine transporter changes (> 50\%) [59]. Consistently, rats with severe denervation (> 75\%) present earlier mitigation by the dopamine transporter, followed by D2R binding changes [60]. Hence, with our method, it is feasible to study

313 the consequences of synaptic dopamine dysfunction without compensations triggered by 314 neuronal cell loss. Moreover, the observed $\left[{ }^{11} \mathrm{C}\right]$ raclopride and $\left[{ }^{11} \mathrm{C}\right] \mathrm{DTBZ}$ correlations to the 315 motor behavior highlight that $\left[{ }^{11} \mathrm{C}\right]$ raclopride binding remains at control levels as long as synaptic dopamine levels are sufficient to maintain adequate motor function. Motor disturbances strongly correlate to pre and postsynaptic changes if movements of the forelimbs, but not whole-body, are considered, in agreement with earlier observations in dopamine-depleted rats [44]. Depletion of VMAT2 resulted in reduced dopamine tissue levels in the ipsilateral striatum, nicely merging with the in vivo data. Moreover, metabolite analysis suggested that due to the lack of VMAT2mediated storage in presynaptic vesicles, dopamine is quickly converted. The increased metabolism might as well be a possible compensatory mechanism consequent to the VMAT2 knockdown, reflecting actions that residual nigrostriatal neurons undertake to maintain dopamine

324 homeostasis, as already speculated by others $[53,61]$.

325 To elucidate the role of VMAT2 in locomotion, reward, and Parkinson`s disease, several 326 investigators have deleted its coding gene in mice [62-65]. Besides the costly and time327 consuming breeding, the gene knockout was not selective for dopaminergic neurons, resulting in 328 the appearance of anxiety and depressive behavior phenotypes [66]. CRISPR/Cas9-editing 329 overcomes these limitations, allowing gene-editing in adult and aged animals and avoiding 
compensatory changes occurring at early developmental stages. Since its discovery, only two been difficult to adapt. Rats are particularly advantageous for imaging studies due to the larger brain size and limited spatial resolution and sensitivity of preclinical scanners [68]. Here, by inducing a mild to moderate gene knockdown, we could investigate early to late resting-state brain network adaptations prompted by presynaptic dysfunction. We show that the selective impairment of presynaptic dopamine storage and release is followed by rs-FC alterations within and between the DMN and SMN. Our results confirm previous findings that the DMN, associated with ideation and mind wandering [69], and the SMN, involved in sensory processing and motor function [70], do not function in isolation from each other, but rather synchronize [71]. The observed internetwork synchronization may reflect compensatory brain reorganization, as already speculated by others $[72,73]$. We also identified enhanced intranetwork rs-FC in the DMN and SMN. Rs-FC changes were observed in prefrontal cortical regions, hippocampus, thalamus, and striatum. Our data parallel previous findings of cortico-striato-thalamic hyperconnectivity in decreased dopamine transmission states [74-77]. In line, increased synchronous neural oscillatory activity and functional coupling in the basal ganglia and its associated networks have been observed in Parkinson`s disease [78-83]. The increase of cortico-striatal FC could in part be due to dysfunctions of multiple tonic inhibitory gate actions of D2R [84]. Increased FC across the thalamus and prefrontal cortex has been reported in drug-treated Parkinson`s disease patients $349[85,86]$, potentially indicating functional compensation, as the brain recruits additional anatomical 350 areas to aid in restoring cognitive processes. This might as well explain the engagement of the 351 hippocampus, functionally connected with DMN cortical regions [87, 88]. In this regard, research 352 has shown that the hyperconnectivity of brain circuits is a common response to neurological 353 dysfunction, and may reflect a protective mechanism to maintain normal brain functioning [89]. 354 Such a mechanism has been proposed in Parkinson`s disease, mild cognitive impairment, and 355 Alzheimer's disease [90-93]. Collectively, our findings support this model and indicate a 
reorganization of brain networks that adapt to the synaptic dysfunction through enhanced interregional synchrony. Recruiting alternative brain regions may be an early response to the dysfunction preceding neuronal cell loss and motor impairment. Interestingly, brain connectome adaptations occurred symmetrically in the DMN but were more weighted towards the contralateral hemisphere in the SMN.

Besides dopamine, dopaminergic neurons co-release GABA via the VMAT2. This hints towards reduced GABA following the VMAT2 knockdown, and consequent imbalance in downstream striatal projection neurons of the direct and indirect pathway [47, 48, 94]. GABA regulates the inhibitory neurotransmission in various brain areas through GABA-A receptors [95]. We hypothesize that decreased binding to the GABA-A receptors may be consistent with loss of inhibitory tone in multiple cortical areas, reflected by our $\left[{ }^{11} \mathrm{C}\right]$ flumazenil PET data, resulting in increased localized brain connectivity, reflected in the fMRI signal. The suppression of the GABAergic feedback circuit, mediated by D2R and external globus pallidus neurons [84], complements the observed elevation in neuronal synchronicity. Our postulation is in line with earlier findings of an inverse correlation of GABA with rs-FC in DMN [96] and with a putative role of loss of inhibitory tone in hyperconnectivity [97]. Interestingly, our $\left[{ }^{11} \mathrm{C}\right]$ flumazenil PET data did not reveal changes in GABA-A expression in the striatum. Instead, we observed a significant decrease in the ipsilateral hemisphere of several cortical regions in both mild and moderate rats,

374 supporting previous findings of GABA modulation of the internetwork FC [98]. Studies report downregulated inhibitory neurotransmission in Parkinson`s disease, where gene expression of

376 GABAergic markers is low in the frontal cortex $[99,100]$. Further, inverse correlations between

$377\left[{ }^{11} \mathrm{C}\right]$ flumazenil binding and gait disturbances [101], and between GABA concentration in the 378 motor cortex and disease severity have been reported [102]. Moreover, our $\left[{ }^{11} \mathrm{C}\right]$ flumazenil PET 379 results suggest that GABA neurotransmission is disturbed already at the mild stage, indicating its 380 potential role as an early biomarker of dopaminergic presynaptic dysfunction. Of note, the 381 observed changes in GABA-A binding might indicate only an apparent decrease in binding due to 
382 the reduced GABA availability, or reflect compensatory adaptation on the contralateral

383 hemisphere. Future research should elucidate these aspects and also perform further

384 investigations on glutamate, due to the crucial role of the excitatory/inhibitory-imbalance in

385 several psychiatric disorders [103].

\section{Limitations and general remarks}

We knocked down VMAT2 in dopaminergic projection neurons from the SNc to the dorsal striatum. To achieve selective targeting of this neuronal subtype, rat Cre driver lines have been developed $[67,104]$. Although all monoamine-releasing neurons express VMAT2, in contrast to other brain regions, SNc neurons are predominantly dopaminergic [105]. Thus, even though we used wild-type rats, we can largely dismiss effects on other monoaminergic neurons, as also indicated by biochemical analysis of serotonin striatal levels.

Despite its limited off-target editing [38], undesired targeting of SaCas9 on other genes cannot be fully excluded. Nevertheless, off-target candidates with up to 4 mismatches were screened in the whole genome of Rattus norvegicus (http://www.rgenome.net/cas-offinder/), consistently with past reports [40]. To the best of our knowledge, the off-target matches (Ndrg1, RGD1305938, Btn2a2, AABR07042293.2) have no effects on VMAT2 function, being involved in cell

400 (https://www.ncbi.nlm.nih.gov/IEB/Research/Acembly/index.html).

401 Another limitation of the study is the relatively small sample size, related to the complex and highcost procedures involved in the in vivo imaging measurements. In addition to this, the intrinsically high intersubject variability in rs-fMRI, and differences in knockdown efficiency contributed to 404 significant variance in our cohorts. Nevertheless, the variability of gene-editing efficiency was in 405 line with previous in vivo brain studies [40, 41]. 
408 This work encourages the combinatorial use of CRISPR/Cas9 and molecular and functional in

409 vivo brain imaging to achieve selective modulation of genes and understand the related functional

410 adaptations in brain networks, beyond the targeted circuitry.

411 We anticipate our approach to be a starting point to shed the light on the function of specific

412 genes and their encoded proteins on whole-brain connectivity, useful to understand the cellular

413 basis of functional changes, identify early neurobiological markers, and promising therapeutic

414 interventions.

415 


\section{Methods}

\section{Animals}

418 Female wild-type Long Evans rats $(224 \pm 30 \mathrm{~g}, \mathrm{n}=57)$ (Charles River Laboratories, Sulzfeld, 419 Germany) were kept on a $12 \mathrm{~h}$ day-night cycle at a room temperature of $22{ }^{\circ} \mathrm{C}$ and $40-60 \%$ 420 humidity. Animals received a standard diet and tap water ad libitum before and during the 421 experimental period. All animal experiments were performed according to the German Animal 422 Welfare Act and were approved by the local ethical authorities, permit numbers R15/19M, 423 R4/20G.

\section{Viral vectors}

SgRNAs targeting the second exon of the S/c18a2 gene and the first exon of the lacZ gene were designed based on the PAM sequence of SaCas9 (NNGRRT) (Table 1). SgRNAs were cloned into an AAV-PHP.EB expression vector containing a GFP reporter sequence, driven by the CMV

429 promoter, for the identification of transduced neurons. A second AAV-PHP.EB construct was 430 produced to express SaCas9, flanked by two nuclear localization sequences (NLS) to allow its 431 translocation into the nuclei. The vector expressed the nuclease via the CAG promoter and 432 contains three HA-tags to visualize the targeted neurons (Fig. 1a). Cloning of the sgRNAs, 433 plasmid construction, as well as the production of concentrated and purified AAV-PHP.EB 434 vectors, delivered at a concentration of $10^{13} \mathrm{gc} / \mathrm{mL}$, were carried by SignaGen Laboratories 435 (Johns Hopkins University, USA). A European patent application has been filed for the AAV436 PHP.EB vectors and is currently pending.

437 The genomic mutation rate was assessed using a different set of AAVs with AAV2/1 serotype, for 438 a conditional design, kindly provided by Matthias Heidenreich (prev. Zhang lab, Broad Institute of 439 MIT and Harvard, Cambridge, USA). 


\section{Rat primary cortical neuron culture}

442 Primary cortical neurons were obtained from rat embryos of a pregnant Sprague Dawley rat from 443 embryonic day 18 (E18) (Charles River, Sulzfeld, Germany). Embryos were decapitated and 444 quickly removed from the mother rat. Cortical dissection was performed in ice-cold HBSS 445 (100 mL $10 \times$ HBSS, $870 \mathrm{~mL} \quad \mathrm{dH}_{2} \mathrm{O}, \quad 3.3 \% \quad 0.3 \mathrm{M}$ HEPES $\mathrm{pH} \quad 7.3$ and $1 \%$ pen/strep) 446 (LifeTechnologies, Massachusetts, USA). The obtained tissue was washed three times with $44710 \mathrm{~mL}$ ice-cold HBSS PhenolRed-free (LifeTechnologies, Massachusetts, USA) and then 448 digested at $37{ }^{\circ} \mathrm{C}$ for $20 \mathrm{~min}$ in $8 \mathrm{~mL} \mathrm{HBSS}$ with $2.5 \%$ trypsin (LifeTechnologies, Massachusetts, 449 USA). Cortices were washed 3 times with $10 \mathrm{~mL}$ HBSS containing $3.7 \% \mathrm{FBS}$, and then gently 450 triturated in $2 \mathrm{~mL}$ HBSS. For the maintenance, neurons were plated on poly-D-lysine-coated 24 451 well plates (BD Biosciences, Heidelberg, Germany) or coverslips (Neuvitro Corporation, 452 Vancouver, USA) at a density of $16 \times 10^{4} /$ well, and cultured in Neurobasal media supplemented 453 with $2 \% 1 \times$ B27, 0.25\% Glutamax, 0.125\% Glutamate and 1\% pen/strep (LifeTechnologies, 454 Massachusetts, USA) for four days. Afterward, for the immunofluorescence, AAVs carrying the 455 expression of the vectors for the SaCas9 and sgRNAs (1:1 ratio), were added to the culture 456 medium at 200,000 MOI (AAV-PHP.EB-sgRNA-lacZ: $1.7 \times 10^{12} \mathrm{gc} / \mathrm{mL}$, AVV-PHP.EB-SaCas9: $457 \quad 1.4 \times 10^{12} \mathrm{gc} / \mathrm{mL}, \quad$ AAV-PHP.EB-sgRNA-S/c18a2: $2.1 \times 10^{12} \mathrm{gc} / \mathrm{mL}, \quad$ final viral volume $45837.5 \mu \mathrm{L} /$ well). For the Surveyor assay, conditional AAVs for SaCas9, sgRNA-Slc18a2, Cre459 recombinase (AAV2/1, 1:1:0.5 ratio) were used. Neurons were processed 1-week post-viral 460 treatment (Fig. 1b).

\section{Surveyor assay}

463 To estimate the VMAT2 knockdown efficiency of the designed sgRNA in vitro, we evaluated the 464 presence of genetic deletions in rat primary neurons with the Surveyor assay (Surveyor kit, 465 Integrated DNA Technologies, Coralville, USA). One week following the viral infection, the 
genomic DNA was extracted using the QuickExtract DNA Extraction solution (Epicentre, Madison, USA), according to the manufacturer's instructions, and was normalized to $100 \mathrm{ng}$ in $\mathrm{dH}_{2}$ O. 18 - 25 nt primers were designed 200 - 400 bp away from either side of the SaCas9 target site to amplify the loci of interest by touchdown PCR (oligonucleotides used for PCR are provided in Supplementary Table 8). DNA amplification was performed using $0.5 \mu \mathrm{L}$ Phusion Polymerase

471 (LifeTechologies, Massachusetts, USA), as previously reported [106]. A single band product was

472 visualized on 1.5\% agarose gel, isolated, and purified using QIAquick Spin columns (Qiagen,

473 Hilden, Germany), following the supplier's protocol. $400 \mathrm{ng}$ of the purified PCR product were

474 mixed with $2 \mu \mathrm{L}$ Taq DNA polymerase buffer (LifeTechnologies, Massachusetts, USA) to allow

475 the cross-annealing of the mutated and wild-type sequences. The re-annealing process was

476 conducted at the following cycling conditions: $10 \mathrm{~min}$ at $95^{\circ} \mathrm{C}, 95^{\circ} \mathrm{C}$ to $85^{\circ} \mathrm{C}$ at $-2{ }^{\circ} \mathrm{C} / \mathrm{s}$, hold

$4771 \mathrm{~min}$, ramp down to $75^{\circ} \mathrm{C}$ at $-0.3^{\circ} \mathrm{C} / \mathrm{s}$, hold $1 \mathrm{~min}$, and so on until $25^{\circ} \mathrm{C}$ temperature was

478 reached. Samples were then stored at $4{ }^{\circ} \mathrm{C}$. This cross-annealing procedure converts the 479 mutations into mismatch duplexes (heteroduplexes), which can be recognized by performing 480 nuclease digestion [107]. We digested the annealed products for $20 \mathrm{~min}$ at $42{ }^{\circ} \mathrm{C}$ using $2.5 \mu \mathrm{L}$ $\mathrm{MgCl}_{2}(0.15 \mathrm{M}), 1 \mu \mathrm{L}$ Surveyor nuclease, and $1 \mu \mathrm{L}$ Surveyor enhancer. Digested products were then resolved on a 2.5\% agarose gel, stained with $0.01 \%$ SYBR Gold DNA (LifeTechnologies, Massachusetts, USA) in 1\% TBE buffer. The size of the occurring bands indicated the location of the mutation (expected DNA fragments sizes are provided in Supplementary Table 8). To quantify the knockdown efficiency, the software ImageJ was used. Peak areas of the bands visualized on agarose gel were selected and the percentages of the transduced neurons acquiring the InDel mutation were calculated using the following formula [106]:

$\operatorname{Editing}(\%)=100 \times\left(1-\sqrt{\left(1-\frac{b+c}{a+b+c}\right)}\right.$ 


\section{Immunofluorescence of rat primary neurons}

492 Rat primary neurons were processed1-week post-AAV-transduction. Coverslips were washed 493 twice with DPBS and fixed in 4\% PFA in DPBS for $15 \mathrm{~min}$ at room temperature. Blocking and 494 permeabilization were performed for $30 \mathrm{~min}$ in DPBS with 5\% donkey serum, 0.5\% Triton-X100, 495 and 0.05\% BSA. Primary antibody incubation (VMAT2, 1:50, EB06558, Everest Biotech, 496 Ramona, USA, HA-tag: 1:50, 682404, Biolegend, San Diego, USA) was performed for 60 min, 497 followed by Cy3 conjugated secondary antibody incubation (1:250, kindly provided from Birgit 498 Fehrenbacher, Department of Dermatology, University of Tuebingen, Germany). Coverslips were 499 mounted using ProLong Antifade Diamond Medium containing DAPI (LifeTechnologies, 500 Massachusetts, USA), and imaged with a TCS-SP2/Leica DM IRE2 confocal laser scanning 501 microscope. Images were processed with Leica Confocal Software LCS (Version 2.61) (original 502 magnification 630) (Leica Microsystems, Wetzlar, Germany).

\section{Study design of the in vivo experiments}

505 In a first cohort, AAVs-CRISPR/SaCas9 were stereotactically delivered into the right SNc of wild-

506 type rats. Afterward, in vivo PET scans with imaging markers of VMAT2 expression, dopamine 507 availability, nerve terminal integrity, and inflammatory responses were performed in VMAT2 508 knockdown and control rats using $\left[{ }^{11} \mathrm{C}\right] \mathrm{DTBZ},\left[{ }^{11} \mathrm{C}\right]$ raclopride, $\left[{ }^{11} \mathrm{C}\right]$ methylphenidate, and $\left[{ }^{18} \mathrm{~F}\right] \mathrm{GE}-$ 509 180, respectively. Motor consequences of the CRISPR/SaCas9-induced VMAT2 knockdown 510 were explored in a wide spectrum of behavioral tasks. Finally, biochemical and histological 511 analyses were performed to corroborate the in vivo data (Fig. 2,3,4a).

512 In a second cohort, cylinder test and $\left[{ }^{11} \mathrm{C}\right]$ raclopride-PET/BOLD-fMRI scans were performed at 513 baseline and after CRISPR/SaCas9-induced VMAT2 knockdown. These measurements were 514 paralleled by in vivo PET scans with imaging markers of VMAT2 and GABA-A expression, to 
515 inspect the extent of the induced VMAT2 knockdown and its impact on GABA signaling, using

$516 \quad\left[{ }^{11} \mathrm{C}\right] \mathrm{DTBZ}$ and $\left[{ }^{11} \mathrm{C}\right]$ flumazenil, respectively (Fig. 5a).

\section{Stereotactic injections}

519 Rats were anesthetized by injecting a mixture $(1 \mathrm{~mL} / \mathrm{kg})$ of fentanyl $(0.005 \mathrm{mg} / \mathrm{kg})$, midazolam

$520(2 \mathrm{mg} / \mathrm{kg}$,) and medetomidine $(0.15 \mathrm{mg} / \mathrm{kg})$ intraperitoneally, and were placed onto a stereotactic

521 frame (Harvard Apparatus, Holliston, MA, USA) with the skull flat between Lambda and Bregma.

522 The following coordinates were used for the injections (flat skull position): AP: $-5 \mathrm{~mm}, \mathrm{ML}$ :

$523 \pm 2 \mathrm{~mm}$, DV: $-7.2 \mathrm{~mm}$, below the dural surface as calculated relative to Bregma. Rats were

524 injected with $3 \mu \mathrm{L}$ AAVs into the right SNc and were divided into two groups. Control rats $(n=10)$

525 were injected with AAV-PHP.EB-sgRNA-lacZ $\left(1.7 \times 10^{12} \mathrm{gc} / \mathrm{mL}\right)$ and AVV-PHP.EB-SaCas9

$526\left(1.4 \times 10^{12} \mathrm{gc} / \mathrm{mL}\right)(1: 1$ ratio $)$. VMAT2 knockdown rats $(n=47)$ were injected with AAV-PHP.EB-

527 sgRNA-S/c18a2 $\left(2.1 \times 10^{12} \mathrm{gc} / \mathrm{mL}\right)$ and AVV-PHP.EB-SaCas9 $\left(1.4 \times 10^{12} \mathrm{gc} / \mathrm{mL}\right)(1: 1 \mathrm{ratio})$ (see

528 Fig. 1a for vector constructs). DPBS (3 $\mu \mathrm{L})$ was sham-injected into the contralateral SNc.

529 Solutions were infused at a rate of $0.2 \mu \mathrm{L} / \mathrm{min}$ using a $5 \mu \mathrm{L}$ Hamilton syringe (Hamilton, Bonaduz,

530 Switzerland) and an automated microsyringe pump (Harvard Apparatus, Holliston, MA, USA). To

531 allow for the diffusion of AAVs into the tissue, the needle was left in place for 10 min, and then

532 slowly retracted at $0.2 \mathrm{~mm} / \mathrm{min}$. After the surgery, an antidote containing atipamezole

$533(0.75 \mathrm{mg} / \mathrm{kg})$ and flumazenil $(0.2 \mathrm{mg} / \mathrm{kg})$ was injected subcutaneously. The rats were kept warm

534 in their cages until fully recovered.

536 Radiotracer synthesis

$537\left[{ }^{11} \mathrm{C}\right] \mathrm{CO}_{2}$ was produced on a medical cyclotron (PETtrace 860, GE Healthcare, Uppsala, 538 Sweden) using the ${ }^{14} \mathrm{~N}(\mathrm{p}, \alpha){ }^{11} \mathrm{C}$ route and converted to either $\left[{ }^{11} \mathrm{C}\right] \mathrm{Mel}$ (methyl iodide) or $539\left[{ }^{11} \mathrm{C}\right] \mathrm{MeOTf}$ (methyl triflate) using a Tracerlab FX Mel module (GE Healthcare). A Tracerlab FX M 
540 module (GE Healthcare) was used for automated methylation, purification, and formulation of the

541 tracers.

$542\left[{ }^{11} \mathrm{C}\right]$ Dihydrotetrabenazine (DTBZ), a VMAT2 ligand, was synthesized starting from $\left[{ }^{11} \mathrm{C}\right] \mathrm{Mel}$,

543 which was reacted with $1 \mathrm{mg}$ (+)-9-O-desmethyl-dihydrotetrabenazine (ABX, Radeberg,

544 Germany) in $300 \mu \mathrm{l} \mathrm{DMF}$ in the presence of $7.5 \mu \mathrm{I} 5 \mathrm{M} \mathrm{NaOH}$ for 3 min at $40{ }^{\circ} \mathrm{C}$ [108]. Afterward,

545 it was purified by HPLC and formulated as a sterile pyrogen-free saline solution. The total

546 synthesis time from the end of the beam was 45 min. The radiochemical purity of the final

547 radiotracer was $>95 \%$ as determined by HPLC. Molar activity at the time of injection was $96 \pm 37$

$548 \mathrm{GBq} / \mathrm{\mu mol}$.

549 D-threo- $\left[{ }^{11} \mathrm{C}\right]$ methylphenidate, a dopamine transporter ligand, was synthesized by alkylation of D550 threo-N-NPS-ritalinic acid (ABX) using $\left[{ }^{11} \mathrm{C}\right]$ Mel [109]. After acidic deprotection, purification, and 551 formulation, the product was obtained with a $37 \pm 6 \%$ decay-corrected radiochemical yield (from $\left.552\left[{ }^{11} \mathrm{C}\right] \mathrm{Mel}\right)$. The total synthesis time was $55 \mathrm{~min}$, and the radiochemical purity of the final 553 formulated radiotracer was $>95 \%$ as determined by HPLC analysis. The molar activity was 554 determined at the time of injection as $58 \pm 14 \mathrm{GBq} / \mu \mathrm{mol}$.

$555\left[{ }^{11} \mathrm{C}\right]$ Raclopride a D2R ligand, was synthesized by alkylation of S-(+)-O-desmethyl-raclopride $556(\mathrm{ABX})$ using $\left[{ }^{11} \mathrm{C}\right] \mathrm{MeOTf}$. After purification and formulation, the product was obtained with a $55712 \pm 4 \%$ decay-corrected radiochemical yield (from $\left[{ }^{11} \mathrm{C}\right]$ methyl triflate). The total synthesis time 558 from the end of the beam was $55 \mathrm{~min}$. The radiochemical purity of the final formulated radiotracer 559 was $>95 \%$ as determined by HPLC. The molar activity was determined at the time of injection 560 and was calculated as $88 \pm 41 \mathrm{GBq} / \mu \mathrm{mol}$.

$561\left[{ }^{11} \mathrm{C}\right]$ Flumazenil, a GABA-A ligand, was synthesized by methylation of desmethylflumazenil (ABX) 562 with $\left[{ }^{11} \mathrm{C}\right] \mathrm{Mel} .2 \mathrm{mg}$ of the precursor were dissolved in $0.3 \mathrm{ml}$ DMF with $3 \mu \mathrm{l} 5 \mathrm{M} \mathrm{NaOH}$ and 563 reacted for $2 \min$ at $80^{\circ} \mathrm{C}$. After the reaction, the product was purified by semi-preparative HPLC 
564 and reformulated by solid-phase extraction (Strata-X, Phenomenex; elution with $0.5 \mathrm{ml}$ ethanol,

565 dilution with $5 \mathrm{ml}$ phosphate-buffered saline). The total synthesis time from the end of the beam

566 was $50 \mathrm{~min}$. The radiochemical purity of the final formulated radiotracer was $>95 \%$ as

567 determined by HPLC. The molar activity was determined at the time of injection as

$568109.5 \pm 39.6 \mathrm{GBq} / \mu \mathrm{mol}$.

$569\left[{ }^{18}\right.$ F]Fluoride was produced on a PETtrace 860 medical cyclotron (GE Healthcare) using 570 the ${ }^{18} \mathrm{O}(\mathrm{p}, \mathrm{n})^{18} \mathrm{~F}$ route.

$571\left[{ }^{18} \mathrm{~F}\right] \mathrm{GE}-180$, a translocator protein ligand [110], was synthesized on a FASTlab synthesizer (GE

572 Healthcare) with precursor and reagent kits in single-use cassettes (GE Healthcare) according to

573 the manufacturer's instructions. The radiochemical purity of the final formulated radiotracer was

$574>95 \%$ as determined by HPLC. The molar activity was determined at the time of injection as

$575576 \pm 283 \mathrm{GBq} / \mu \mathrm{mol}$.

577 In vivo PET imaging and data analysis

578 For the study of cohort 1, VMAT2 knockdown $(n=14)$ and control rats $(n=10)$ underwent 60 min

579 dynamic PET emission scans with $\left[{ }^{11} \mathrm{C}\right] \mathrm{DTBZ}$ (8 - 10 weeks post-injection), $\left[{ }^{11} \mathrm{C}\right]$ methylphenidate

580 (10 - 12 weeks post-injection), $\left[{ }^{11} \mathrm{C}\right]$ raclopride $\left(12-14\right.$ weeks post-injection) and $\left[{ }^{18} \mathrm{~F}\right] \mathrm{GE}-180$

581 (14 - 16 weeks post-injection) (Fig. 2a). Four rats were excluded from the data analyses because

582 two rats from each group died during a PET acquisition. One control rat was excluded from the

$583\left[{ }^{11} \mathrm{C}\right] \mathrm{methylphenidate}$ analysis due to a poor signal-to-noise ratio.

584 For the study of cohort 2, VMAT2 knockdown rats $(n=33)$, underwent 60 min dynamic PET 585 emission acquisitions with $\left[{ }^{11}\right.$ C]DTBZ (8 - 10 weeks post-injection), and $\left[{ }^{11} \mathrm{C}\right]$ flumazenil $(10-$ 58612 weeks post-injection). The final cohort included 23 rats (see paragraph Simultaneous 587 PET/fMRI experiments). 
Three small-animal PET scanners (Inveon, Siemens, Erlangen, Germany) and dedicated rat brain beds (Jomatik Gmbh, Tuebingen, Germany) with stereotactic holders and temperature feedback control units (Medres, Cologne, Germany) were used. These ensured the delivery and removal of the anesthesia gas and stabilized the body temperature at $37^{\circ} \mathrm{C}$ during the PET data acquisition. Anesthesia was induced by placing the animals in knock-out boxes and delivering $2 \%$ isoflurane in oxygen air. Subsequently, a 24 G catheter (BD Insyte, NJ, USA) was placed into the tail vein for the tracer and/or i.v. anesthesia administration. Afterward, animals of cohort 1 were anesthetized with $2 \%$ isoflurane vaporized in $1.0 \mathrm{~L} / \mathrm{min}$ of oxygen. Animals of cohort 2 received a medetomidine bolus injection $(0.05 \mathrm{mg} / \mathrm{kg})$ and the anesthesia was switched to constant medetomidine infusion $(0.1 \mathrm{mg} / \mathrm{kg} / \mathrm{h})$, and $0.5 \%$ isoflurane in air during the scan time, as adapted

598 from the literature [111].

599 The rats were placed in the center of the field of view and PET acquisitions started $5 \mathrm{~s}$ before the 600 bolus injection of the tracer. In Supplementary Tables 9 and 10, injected activity (MBq/kg) and molar activity $(\mathrm{GBq} / \mu \mathrm{mol})$ at the time of injection are reported for each radioligand. The list-mode data from the dynamic acquisitions of $\left[{ }^{11} \mathrm{C}\right] \mathrm{DTBZ},\left[{ }^{11} \mathrm{C}\right]$ raclopride, $\left[{ }^{11} \mathrm{C}\right]$ methylphenidate, and $\left[{ }^{11} \mathrm{C}\right]$ flumazenil were histogrammed into 39 time-frames $(12 \times 5 \mathrm{~s}, 6 \times 10 \mathrm{~s}, 6 \times 30 \mathrm{~s}, 5 \times 60 \mathrm{~s}$, $10 \times 300$ s), from $\left[{ }^{18} \mathrm{~F}\right] \mathrm{GE}-180$ into 16 time-frames (5x60 s, 5x120 s, 3x300 s, 3x600 s). PET scans of the study cohort 1 were reconstructed using the OSEM3D map algorithm, and a matrix size of $256 \times 256 \times 159$, resulting in a pixel size of $0.38 \times 0.38 \times 0.79 \mathrm{~mm}$. PET scans of the study cohort 2 were reconstructed using the OSEM2D algorithm, and a matrix size of $256 \times 256 \times 89$, resulting 608 in a pixel size of $0.33 \times 0.33 \times 0.79 \mathrm{~mm}$.

609 Data preprocessing analysis was performed with Matlab (Mathworks, Natick, MA, USA), 610 Statistical Parametric Mapping 12 (SPM12, Wellcome Trust Centre for Neuroimaging, University 611 College London, England), and the QModeling toolbox [112]. First, realignment of all frames was 612 performed using SPM12 and average images were generated for every scan. The mean images 613 were then used for coregistration to the Schiffer rat brain atlas provided by PMOD software [113]. 
614 To generate the respective time activity curves (TAC), volumes of interest (VOIs) were defined

615 over the target and reference regions. VOIs were placed over the right and left striatum for

$616\left[{ }^{11} \mathrm{C}\right] \mathrm{DTBZ},\left[{ }^{11} \mathrm{C}\right]$ raclopride, and $\left[{ }^{11} \mathrm{C}\right]$ methylphenidate, and over the regions reported in Table 2 for

$617\left[{ }^{11} \mathrm{C}\right]$ flumazenil. Cerebellum was used as reference region for $\left[{ }^{11} \mathrm{C}\right] \mathrm{DTBZ},\left[{ }^{11} \mathrm{C}\right] \mathrm{raclopride}$ and

$618\left[{ }^{11} \mathrm{C}\right]$ methylphenidate. Pons was used as reference region for $\left[{ }^{11} \mathrm{C}\right]$ flumazenil.

619 Binding potentials for $\left[{ }^{11} \mathrm{C}\right] \mathrm{DTBZ},\left[{ }^{11} \mathrm{C}\right]$ raclopride, $\left[{ }^{11} \mathrm{C}\right]$ methylphenidate and $\left[{ }^{11} \mathrm{C}\right]$ flumazenil were

620 calculated over the all frames in the regions of interest with Logan reference [114], with a

621 population average k2' ([11 C]DTBZ: $0.41 \mathrm{~min}^{-1},\left[{ }^{11} \mathrm{C}\right]$ raclopride: $0.34 \mathrm{~min}^{-1},\left[{ }^{11} \mathrm{C}\right]$ methylphenidate:

$6220.18 \mathrm{~min}^{-1},\left[{ }^{11} \mathrm{C}\right]$ flumazenil: $\left.0.27 \mathrm{~min}^{-1}\right) .\left[{ }^{11} \mathrm{C}\right] \mathrm{DTBZ}$ and $\left[{ }^{11} \mathrm{C}\right]$ raclopride binding changes (\%), here

623 expressed as $\Delta$ binding, were calculated according to the formula:

$\Delta$ binding $=\frac{1-\text { binding right striatum }}{\text { binding left striatum }} \times 100$

$624\left[{ }^{18} \mathrm{~F}\right] \mathrm{GE}-180$ uptake in the right striatum was calculated over the interval between $30-60 \mathrm{~min}$

625 after scan start and normalized by the uptake calculated in the left (DPBS-injected) striatum over

626 the same time interval.

627 QModeling was used to generate voxel-wise binding potential maps for $\left[{ }^{11} \mathrm{C}\right] \mathrm{DTBZ}$,

$628\left[{ }^{11} \mathrm{C}\right]$ raclopride, $\left[{ }^{11} \mathrm{C}\right]$ methylphenidate, and $\left[{ }^{11} \mathrm{C}\right]$ flumazenil. $\left[{ }^{18} \mathrm{~F}\right] \mathrm{GE}-180$ average uptake images

629 were generated with an in-house-written script in MATLAB.

630

\section{Simultaneous PET/fMRI experiments}

632 Rats $(n=33)$ underwent longitudinal simultaneous $\left[{ }^{11} \mathrm{C}\right]$ raclopride-PET/BOLD-fMRI scans at 633 baseline and $8-14$ weeks after CRISPR/SaCas9-induced VMAT2 knockdown. Three rats had to 634 be excluded from the data analyses due to aliasing artifacts, local distortion, and motion during 635 the data acquisition. Seven rats died during a PET/BOLD-fMRI scan. The final cohort included 23 
rats. Anesthesia induction and injection were performed as described above for the cohort 2 (see paragraph In vivo PET imaging and data analysis).

638 Next, rats were placed onto a dedicated rat bed (Medres, Cologne, Germany) and a temperature 639 feedback control unit (Medres, Cologne, Germany), ensuring the delivery and removal of the 640 anesthesia gas and stabilizing the body temperature at $37^{\circ} \mathrm{C}$ during the scan time. A breathing 641 pad and a pulse oximeter were used to observe respiration and heart rates. Scans were acquired 642 using a small-animal $7 \mathrm{~T}$ Clinscan MRI scanner, a $72 \mathrm{~cm}$ diameter linearly polarized RF coil 643 (Bruker) for transmission, and a four-channel rat brain coil for reception (Bruker Biospin MRI, 644 Ettlingen, Germany). Localizer scans were first acquired to accurately position the rat brains into 645 the center of the PET/MRI field of view. Subsequently, local field homogeneity was optimized by 646 measuring local magnetic field maps. Anatomical reference scans were performed using T2647 weighted Turbo-RARE MRI sequences (TR: $1800 \mathrm{~ms}$, TE: $67.11 \mathrm{ms,} \mathrm{FOV:} 40 \times 32 \times 32 \mathrm{~mm}$, 648 image size: $160 \times 128 \times 128$ px, Rare factor: 28, averages: 1). Finally, T2*-weighted gradient echo EPI sequences (TR: $2500 \mathrm{~ms}$, TE: $18 \mathrm{~ms}, 0.25 \mathrm{~mm}$ isotropic resolution, FoV 25 x $23 \mathrm{~mm}$, image size: $92 \times 85 \times 20$ px, slice thickness $0.8 \mathrm{~mm}$, slice separation $0.2 \mathrm{~mm}$, 20 slices) were acquired for BOLD-fMRI.

652 A dedicated small-animal PET insert developed in cooperation with Bruker (Bruker Biospin MRI, 653 Ettlingen, Germany) was used for the $\left[{ }^{11} \mathrm{C}\right]$ raclopride acquisitions, the second generation of a 654 PET insert developed in-house with similar technical specifications [115]. $\left[{ }^{11} \mathrm{C}\right]$ Raclopride was 655 applied via a bolus injection. In Supplementary Table 10 injected activities (MBq/kg) and molar 656 activities $(\mathrm{GBq} / \mu \mathrm{mol})$ at the time of injection are reported. PET/fMRI acquisitions started 657 simultaneously with the tracer injection and were performed over a period of $60 \mathrm{~min}$. The list658 mode files of the PET data were histogrammed into 14 time-frames $(1 \times 30 \mathrm{~s}, 5 \times 60 \mathrm{~s}, 5 \times 300 \mathrm{~s}$, $6593 \times 600 \mathrm{~s})$, the 30s between acquisition start and the injection were excluded from the analysis. 660 Reconstruction was performed with an in-house-written OSEM2D algorithm. Data preprocessing 661 and analysis were performed as described above (see paragraph In vivo PET imaging and data 
analysis). A population average $\mathrm{k} 2$ ' $\left(\left[{ }^{11} \mathrm{C}\right]\right.$ raclopride baseline: $0.20 \mathrm{~min}^{-1},\left[{ }^{11} \mathrm{C}\right]$ raclopride VMAT2

663 knockdown: $0.23 \mathrm{~min}^{-1}$ ) was set for the Logan reference [114].

664 Preprocessing of the fMRI data was performed using a pipeline employing SPM12, Analysis of 665 Functional Neurolmages (AFNI, National Institute of Mental Health (NIMH), Bethesda, Maryland, 666 USA), and in-house-written scripts in MATLAB, as reported previously [116].

667 RS-FC was calculated using a seed-based approach. To this extent, 20 regions were selected 668 from the Schiffer rat brain atlas (a list of the regions is provided in Table 2). The SPM toolbox 669 Marseille Boîte À Région d'Intérêt (MarsBaR) was employed to extract fMRI time-courses from all 670 regions [117]. These were then used to calculate pairwise Pearson's $r$ correlation coefficients for 671 each dataset, generating correlation matrices containing $20 \times 20$ elements. Self-correlations were 672 set to zero. The computed Pearson's $r$ coefficients then underwent Fischer's transformation into $z$ 673 values for group-level analysis.

674 Several rs-FC metrics were computed on different regional levels to investigate the potential 675 effects of dopamine depletion in the right striatum. Regional node strengths were computed as 676 the sum of all correlations of one seed to the regions included in one network. Interregional node 677 strengths were defined as the sum of the correlations of one node to the regions of another 678 network. Network strengths were defined as the sum of strengths of all correlations between 679 regions belonging to a network. Internetwork strengths were calculated as the sum of all 680 correlations between two sets of regions belonging to two networks [118].

\section{Behavioral analysis} started immediately and lasted 5 min. During the test session rats were left undisturbed and were videotaped with a camera located at the bottom-center of the cylinder to allow a $360^{\circ}$ angle view. 
number of wall touches, contacts with fully extended digits, was counted. Data were analyzed as

follows:

contralateral paw touches $(\%$ to total $)=\frac{\text { contralateral touches }}{\text { total touches }} \times 100$

690 One rat from the cohort 2 was excluded from the analysis due to issues during video recording.

\section{Rotameter test}

Rotational asymmetry was assessed using an automated rotameter system composed of four

694 hemispheres (TSE Systems GmBH, Bad Homburg, Germany) based on the design of Ungerstedt

695 and Arbuthnott [119]. Rats were placed into an opaque half bowl (49 cm $\varnothing ; 44 \mathrm{~cm}$ height) and

696 fixed to a moveable wire with a collar. The number of clockwise (CW) and counterclockwise

697 (CCW) rotations (difference of $42.3^{\circ}$ in their position) were automatically counted. The

698 spontaneous rotation test lasted $5 \mathrm{~min}$. Apomorphine-evoked rotational asymmetry was

699 evaluated for $60 \mathrm{~min}$ after s.c. administration of apomorphine hydrochloride $(0.25 \mathrm{mg} / \mathrm{kg})$

700 dissolved in physiological saline containing $0.1 \%$ ascorbic acid (Sigma Aldrich, St. Louis,

701 Missouri, USA). Two priming injections of apomorphine (1-week interval off-drug) were necessary

702 to produce sensitization to the treatment. The program RotaMeter (TSE Systems $\mathrm{GmBH}$, Bad

703 Homburg, Germany) was used to acquire the data. Data were analyzed as follows:

turns $/ \mathrm{min}=c c w$ rotation $-c w$ rotations

Beam walk test

Rats were trained for 4 days to cross a built-in-house beam $(1.7 \mathrm{~cm}$ width, $60 \mathrm{~cm}$ length, $40 \mathrm{~cm}$ height), and reach a cage with environmental enrichment. Rats had 5 trials to cross the beam with the reward resting time decreasing from $30 \mathrm{~s}$ to $10 \mathrm{~s}$. On the test day (one week apart from the fourth day of training), rats were videotaped. The acquired videos were analyzed using a 

counted. Data were analyzed as follows:

contralateral footslips $(\%$ to total steps $)=\frac{\text { contralateral footslips }}{\text { total steps }} \times 100$

714 Untrained rats were set in a rectangular box (TSE Systems GmBH, Bad Homburg, Germany) for

$71511 \mathrm{~min}$ (1 $\mathrm{min}$ habituation, $10 \mathrm{~min}$ test) to evaluate the spontaneous exploratory behavior. A

716 frame with light sensors, as high as the animals' body center, was connected to a receiver box to

717 record the rats' walked distance. The program ActiMod (TSE Systems GmBH, Bad Homburg,

718 Germany) was used for the analysis and the experimental session was divided into 1 min time

719 bins. Results were averaged from the total traveled distance over 10 min (mean \pm SD).

Rats' body weight was measured before (week 0) and 14 weeks after CRISPR/SaCas9-induced

VMAT2 knockdown. Body weight gain was calculated as follows:

body weight gain $(g)=\frac{\text { weight at week } 14-\text { weight at week } 0}{\text { weight at week } 0} \times 100$

\section{Histology of rat brain slices}

Rats of cohort $1(n=20)$ were sacrificed 19 weeks after viral vector injection via $\mathrm{CO}_{2}$ inhalation brains were rapidly removed and placed in a brain matrix on ice. The left and right striata were 
731 and then transferred to a $20 \%$ sucrose solution for cryoprotection. Brains were cut into $35 \mu \mathrm{m}$

732 thick coronal sections on a freezing microtome (Leica Biosystems, Wetzlar, Germany) and stored

733 in an anti-freeze solution (0.5 M phosphate buffer, $30 \%$ glycerol, $30 \%$ ethylene glycol) at -

$73420^{\circ} \mathrm{C}$. Sections were collected in 12 equally spaced series through the entire anterior-posterior

735 extent of the $\mathrm{SN}$ and striatum and stored until further analysis. Immunohistochemistry was

736 performed on free-floating sections. Sections were washed 3 times with TBS buffer and antigen

737 retrieval was carried for $30 \mathrm{~min}$ at $80^{\circ} \mathrm{C}$ in Tris/EDTA buffer. Afterward, pre-incubation in $\mathrm{MeOH}$

738 (10\%) and $\mathrm{H}_{2} \mathrm{O}_{2}(3 \%)$ in TBS was performed for 30 min. Following the blocking in 5\% normal

739 goat serum in TBS-X (0.05\%), primary antibody incubation was performed for $24 \mathrm{~h}$ at room

740 temperature in 1\% BSA in TBS-X (TH: 1:5000, P40101, Pel-freez, Arkansas, USA, VMAT2:

741 1:5000, 20042, Immunostar, Hudson, USA). The tissue was rinsed in TBS-X and reacted with the

742 respective biotinylated secondary antibody (1:200, Vector Laboratories Ltd., Peterborough, UK)

743 for $60 \mathrm{~min}$ at room temperature in 1\% BSA in TBS-X. Staining was developed using 3,3'-

744 diaminobenzidine (DAB Substrate Kit, Vector Laboratories Ltd., Peterborough, UK) and an

745 immunoperoxidase system (Vectastain Elite ABC-Kit, Vector Laboratories Ltd., Peterborough,

746 UK). Slices were rinsed, mounted onto chromalum gelatinized slides, dehydrated in ascending

747 concentrations of alcohol and xylene baths, and coverslipped with DPX mounting medium (Sigma

748 Aldrich, St. Louis, Missouri, USA).

749

750 Stereological analysis

751 Estimates of total numbers of $\mathrm{TH}+$ cells in nigral sections were obtained with an unbiased

752 stereological quantification method by employing the optical fractionator principle [120]. Brain

753 sections from 5 rats (VMAT2 knockdown $n=2$, Control $n=3$ ) were excluded from the stereological

754 analysis, due to weak TH immunoreactivity. First, 5x images were acquired with the automated

755 Metafer slide scanning platform (MetaSystems, Altlußheim, Germany). Then, ROIs were drawn

756 using the VSViewer program (Metasystems, Altlußheim, Germany), and a sampling fraction of 
$75750 \%$ was defined. Afterward, 63x images were acquired in an automated fashion based on the

758 sampling fraction in a random orientation within the ROI. The acquired 63x images were imported

759 into the VIS program and cell counting was performed with the CAST module (Visiopharm A/S,

760 Hørsholm, Denmark, Version 2020.08.2.8800).

761 The number of cells estimates was obtained by applying the formula:

Total number of $\mathrm{TH}+$ cells $=\sum\left(\frac{\text { Number of cells counted per brain }}{\text { Acquisition fraction } x \text { counting frame }}\right) \times$ number of series

Immunofluorescence of rat brain slices

Immunofluorescence was performed on SN sections from rats of cohort 1 (35 $\mu \mathrm{m}$ thick coronal

sections), mounted onto chromalum gelatinized slides, dehydrated in ascending concentrations of alcohol and xylene baths. Sections were washed 3 times with KPBS buffer and antigen retrieval was carried for $30 \mathrm{~min}$ at $80{ }^{\circ} \mathrm{C}$ in Tris/EDTA buffer. Following blocking in $5 \%$ donkey serum and normal horse serum in KPBS-X (0.25\%), primary antibody incubation was performed for $24 \mathrm{~h}$ at room temperature (VMAT2: 1:5000, 20042, Immunostar, Hudson, USA, HA-tag: 1:5000, MMS101R, Nordic BioSite, Taby, Sweden, GFP: 1:50.000, ab13970, Abcam, Cambridge, UK). The tissue was rinsed in KPBS-X and reacted with the respective fluorophore-conjugated secondary antibody (1:200, Vector Laboratories Ltd., Peterborough, UK) for $2 \mathrm{~h}$ in $0.2 \%$ KPBS-X. Slides were coverslipped with Vectashield mounting medium (Vector Laboratories Ltd., Peterborough, 774 UK).

777 Dopamine, 3,4-Dihydroxyphenylacetic acid (DOPAC), homovanillic acid (HVA), and serotonin (5$778 \mathrm{HT}$ ) striatal levels were determined by HPLC. Brain samples from 5 rats (VMAT2 knockdown 
$779 n=3$, Control $n=2$ ) were excluded from the analysis as striatal sections did not fall in the selected

780 range: $1.2-0.4 \mathrm{~mm}$.

781 Briefly, striatal brain lysates generated from cohort 1 were injected by a cooled autosampler into an ESA Coulchem III coupled to a Decade Elite electrochemical detector (Antec Scientific, Zoeterwoude, The Netherlands) set to a potential of $+350 \mathrm{mV}$. Separation was facilitated by using an Atlantis Premier BEH C18 AX column (Waters Corporation, Massachusetts, USA) and a dual mobile phase gradient of decreasing octane sulfonic acid (OSA) and increasing MeOH content

786 (mobile phase A containing $100 \mathrm{mM} \mathrm{PO}_{4}$-buffer $\mathrm{pH} 2.50$ and $4.62 \mathrm{mM}$ OSA and mobile phase $\mathrm{B}$ 787 containing $100 \mathrm{mM} \mathrm{PO}_{4}$-buffer $\mathrm{pH} 2.50$ and $2.31 \mathrm{mM}$ OSA), delivered at a flow rate of $7880.35 \mathrm{~mL} / \mathrm{min}$ to an Atlantis Premier BEH C18 column (particle size $2.5 \mu \mathrm{m}, 2.1 \mathrm{~mm} \times 150 \mathrm{~mm}$ ) 789 (Waters Corporation, Massachusetts, USA). Data was collected using the Chemstation software 790 (Agilent, California, USA) and then exported to Chromeleon (LifeTechnologies, Massachusetts, 791 USA) for data quality control, peak integration, and concentration calculations. Striatal 792 metabolites' content was expressed in nmol for each sample and normalized to total protein (mg).

793 Dopamine turnover rate was calculated according to the formula:

Dopamine turnover rate $=\frac{H V A+D O P A C}{D A}$

Statistics

Statistical analysis was performed with GraphPad Prism 9.0 (Graphpad Software) if not otherwise

797 stated. Results were analyzed using paired t-tests for the within-subjects comparisons, and 798 unpaired t-tests for the between-groups comparisons. Correlations were performed using linear regression analyses. Synaptic dysfunction discrimination was tested with multiple-comparison ANOVA. 


\section{Bibliography}

803 1. Biswal, B., et al., Functional connectivity in the motor cortex of resting human brain using echoplanar MRI. Magn Reson Med, 1995. 34(4): p. 537-41.

2. Ogawa, S., et al., Brain magnetic resonance imaging with contrast dependent on blood oxygenation. Proc Natl Acad Sci U S A, 1990. 87(24): p. 9868-72.

3. Lu, H., et al., Rat brains also have a default mode network. Proc Natl Acad Sci U S A, 2012. 109(10): p. 3979-84.

4. Becerra, L., et al., Robust reproducible resting state networks in the awake rodent brain. PLoS One, 2011. 6(10): p. e25701.

5. Fox, M.D., et al., The human brain is intrinsically organized into dynamic, anticorrelated functional networks. Proc Natl Acad Sci U S A, 2005. 102(27): p. 9673-8.

6. Jonckers, E., et al., Functional connectivity $f M R I$ of the rodent brain: comparison of functional connectivity networks in rat and mouse. PLoS One, 2011. 6(4): p. e18876.

7. Wehrl, H.F., et al., Simultaneous PET-MRI reveals brain function in activated and resting state on metabolic, hemodynamic and multiple temporal scales. Nat Med, 2013. 19(9): p. 1184-9.

8. Amend, M., et al., Functional resting-state brain connectivity is accompanied by dynamic correlations of application-dependent [(18)F]FDG PET-tracer fluctuations. Neuroimage, 2019. 196: p. 161-172.

9. Ionescu, T.M., et al., Elucidating the complementarity of resting-state networks derived from dynamic [(18)F]FDG and hemodynamic fluctuations using simultaneous small-animal PET/MRI. Neuroimage, 2021. 236: p. 118045.

10. Badhwar, A., et al., Resting-state network dysfunction in Alzheimer's disease: A systematic review and meta-analysis. Alzheimers Dement (Amst), 2017. 8: p. 73-85.

11. Bluhm, R.L., et al., Spontaneous low-frequency fluctuations in the BOLD signal in schizophrenic patients: anomalies in the default network. Schizophr Bull, 2007. 33(4): p. 1004-12.

12. Volkow, N.D., et al., Imaging endogenous dopamine competition with [11C]raclopride in the human brain. Synapse, 1994. 16(4): p. 255-62.

13. Laruelle, M., Imaging synaptic neurotransmission with in vivo binding competition techniques: $a$ critical review. J Cereb Blood Flow Metab, 2000. 20(3): p. 423-51.

14. Patel, V.D., et al., Imaging dopamine release with Positron Emission Tomography (PET) and (11)Craclopride in freely moving animals. Neuroimage, 2008. 41(3): p. 1051-66.

15. Roffman, J.L., et al., Dopamine D1 signaling organizes network dynamics underlying working memory. Science advances, 2016. 2(6): p. e1501672-e1501672.

16. McCutcheon, R.A., et al., Mesolimbic Dopamine Function Is Related to Salience Network Connectivity: An Integrative Positron Emission Tomography and Magnetic Resonance Study. Biol Psychiatry, 2019. 85(5): p. 368-378.

17. Nagano-Saito, A., et al., Posterior dopamine D2/3 receptors and brain network functional connectivity. Synapse, 2017. 71(11).

18. Hahn, A., et al., Differential modulation of the default mode network via serotonin-1A receptors. Proc Natl Acad Sci U S A, 2012. 109(7): p. 2619-24.

19. Vidal, B., et al., In vivo biased agonism at 5-HT(1A) receptors: characterisation by simultaneous PET/MR imaging. Neuropsychopharmacology, 2018. 43(11): p. 2310-2319.

20. Shiyam Sundar, L.K., et al., Fully Integrated PET/MR Imaging for the Assessment of the Relationship Between Functional Connectivity and Glucose Metabolic Rate. Front Neurosci, 2020. 14: p. 252.

21. Sander, C.Y., et al., Imaging Agonist-Induced D2/D3 Receptor Desensitization and Internalization In Vivo with PET/fMRI. Neuropsychopharmacology, 2016. 41(5): p. 1427-36. 
849

850

851

852

853

854

855

856

857

858

859

860

861

862

863

864

865

866

867

868

869

870

871

872

873

874

875

876

877

878

879

880

881

882

883

884

885

886

887

888

889

890

891

892

893

894

895

896

22. Ionescu, T.M., et al., Striatal and prefrontal D2R and SERT distributions contrastingly correlate with default-mode connectivity. Neuroimage, 2021. 243: p. 118501.

23. Betley, J.N. and S.M. Sternson, Adeno-associated viral vectors for mapping, monitoring, and manipulating neural circuits. Hum Gene Ther, 2011. 22(6): p. 669-77.

24. Jinek, M., et al., RNA-programmed genome editing in human cells. Elife, 2013. 2: p. e00471.

25. Cong, L., et al., Multiplex genome engineering using CRISPR/Cas systems. Science, 2013. 339(6121): p. 819-23.

26. Whitworth, K.M., et al., Use of the CRISPR/Cas9 system to produce genetically engineered pigs from in vitro-derived oocytes and embryos. Biol Reprod, 2014. 91(3): p. 78.

27. Chen, Y., et al., Functional disruption of the dystrophin gene in rhesus monkey using CRISPR/Cas9. Hum Mol Genet, 2015. 24(13): p. 3764-74.

28. Chang, N., et al., Genome editing with RNA-guided Cas9 nuclease in zebrafish embryos. Cell Res, 2013. 23(4): p. 465-72.

29. Platt, R.J., et al., CRISPR-Cas9 knockin mice for genome editing and cancer modeling. Cell, 2014. 159(2): p. 440-55.

30. Yu, Z., et al., Highly efficient genome modifications mediated by CRISPR/Cas9 in Drosophila. Genetics, 2013. 195(1): p. 289-91.

31. Musunuru, K., et al., In vivo CRISPR base editing of PCSK9 durably lowers cholesterol in primates. Nature, 2021. 593(7859): p. 429-434.

32. Ousterout, D.G., et al., Multiplex CRISPR/Cas9-based genome editing for correction of dystrophin mutations that cause Duchenne muscular dystrophy. Nat Commun, 2015. 6: p. 6244.

33. Swiech, L., et al., In vivo interrogation of gene function in the mammalian brain using CRISPRCas9. Nature Biotechnology, 2015. 33(1): p. 102-U286.

34. Wilbie, D., J. Walther, and E. Mastrobattista, Delivery Aspects of CRISPR/Cas for in Vivo Genome Editing. Accounts of chemical research, 2019. 52(6): p. 1555-1564.

35. Wu, W.Y., et al., Genome editing by natural and engineered CRISPR-associated nucleases. Nat Chem Biol, 2018. 14(7): p. 642-651.

36. Zetsche, B., et al., Cpf1 is a single RNA-guided endonuclease of a class 2 CRISPR-Cas system. Cell, 2015. 163(3): p. 759-71.

37. Kleinstiver, B.P., et al., Engineered CRISPR-Cas9 nucleases with altered PAM specificities. Nature, 2015. 523(7561): p. 481-5.

38. Ran, F.A., et al., In vivo genome editing using Staphylococcus aureus Cas9. Nature, 2015. 520(7546): p. 186-91.

39. Nishimasu, H., et al., Crystal Structure of Staphylococcus aureus Cas9. Cell, 2015. 162(5): p. 111326.

40. Sun, H., et al., Development of a CRISPR-SaCas9 system for projection- and function-specific gene editing in the rat brain. Sci Adv, 2020. 6(12): p. eaay6687.

41. Kumar, N., et al., The Development of an AAV-Based CRISPR SaCas9 Genome Editing System That Can Be Delivered to Neurons in vivo and Regulated via Doxycycline and Cre-Recombinase. Front Mol Neurosci, 2018. 11: p. 413.

42. Hunker, A.C., et al., Conditional Single Vector CRISPR/SaCas9 Viruses for Efficient Mutagenesis in the Adult Mouse Nervous System. Cell Rep, 2020. 30(12): p. 4303-4316 e6.

43. Liu, Y. and R.H. Edwards, The role of vesicular transport proteins in synaptic transmission and neural degeneration. Annu Rev Neurosci, 1997. 20: p. 125-56.

44. Miyanishi, K., et al., Behavioral tests predicting striatal dopamine level in a rat hemi-Parkinson's disease model. Neurochem Int, 2019. 122: p. 38-46.

45. Tomasi, D., et al., Dopamine transporters in striatum correlate with deactivation in the default mode network during visuospatial attention. PLoS One, 2009. 4(6): p. e6102. 
46. Sambataro, F., et al., DRD2 genotype-based variation of default mode network activity and of its relationship with striatal DAT binding. Schizophr Bull, 2013. 39(1): p. 206-16.

47. Tritsch, N.X., J.B. Ding, and B.L. Sabatini, Dopaminergic neurons inhibit striatal output through non-canonical release of GABA. Nature, 2012. 490(7419): p. 262-6.

48. Tritsch, N.X., et al., Midbrain dopamine neurons sustain inhibitory transmission using plasma membrane uptake of GABA, not synthesis. Elife, 2014. 3: p. e01936.

49. Chatterjee, D., et al., Enhanced CNS transduction from AAV.PHP.eB infusion into the cisterna magna of older adult rats compared to AAV9. Gene Ther, 2021.

50. Hume, S.P., et al., Effect of L-dopa and 6-hydroxydopamine lesioning on [11C]raclopride binding in rat striatum, quantified using PET. Synapse, 1995. 21(1): p. 45-53.

51. Mishra, R.K., A.M. Marshall, and S.L. Varmuza, Supersensitivity in rat caudate nucleus: effects of 6hydroxydopamine on the time course of dopamine receptor and cyclic AMP changes. Brain Res, 1980. 200(1): p. 47-57.

52. Creese, I., D.R. Burt, and S.H. Snyder, Dopamine receptor binding enhancement accompanies lesion-induced behavioral supersensitivity. Science, 1977. 197(4303): p. 596-8.

53. Konieczny, J., et al., The significance of rotational behavior and sensitivity of striatal dopamine receptors in hemiparkinsonian rats: A comparative study of lactacystin and 6-OHDA. Neuroscience, 2017. 340: p. 308-318.

54. Wang, Y.M., et al., Knockout of the vesicular monoamine transporter 2 gene results in neonatal death and supersensitivity to cocaine and amphetamine. Neuron, 1997. 19(6): p. 1285-96.

55. Seeman, P., et al., Dopamine receptors in the central nervous system. Fed Proc, 1978. 37(2): $\mathrm{p}$. 131-6.

56. Gnanalingham, K.K. and R.G. Robertson, The effects of chronic continuous versus intermittent levodopa treatments on striatal and extrastriatal D1 and D2 dopamine receptors and dopamine uptake sites in the 6-hydroxydopamine lesioned rat--an autoradiographic study. Brain Res, 1994. 640(1-2): p. 185-94.

57. Graham, W.C., A.R. Crossman, and G.N. Woodruff, Autoradiographic studies in animal models of hemi-parkinsonism reveal dopamine D2 but not D1 receptor supersensitivity. I. 6-OHDA lesions of ascending mesencephalic dopaminergic pathways in the rat. Brain Res, 1990. 514(1): p. 93-102.

58. Antonini, A., et al., Long-term changes of striatal dopamine D2 receptors in patients with Parkinson's disease: a study with positron emission tomography and [11C]raclopride. Mov Disord, 1997. 12(1): p. 33-8.

59. Kraemmer, J., et al., Correlation of striatal dopamine transporter imaging with post mortem substantia nigra cell counts. Mov Disord, 2014. 29(14): p. 1767-73.

60. Sossi, V., et al., Dopamine transporter relation to levodopa-derived synaptic dopamine in a rat model of Parkinson's: an in vivo imaging study. J Neurochem, 2009. 109(1): p. 85-92.

61. Zigmond, M.J., T.G. Hastings, and R.G. Perez, Increased dopamine turnover after partial loss of dopaminergic neurons: compensation or toxicity? Parkinsonism Relat Disord, 2002. 8(6): p. 38993.

62. Takahashi, N., et al., VMAT2 knockout mice: heterozygotes display reduced amphetamineconditioned reward, enhanced amphetamine locomotion, and enhanced MPTP toxicity. Proc Natl Acad Sci U S A, 1997. 94(18): p. 9938-43.

63. Fon, E.A., et al., Vesicular transport regulates monoamine storage and release but is not essential for amphetamine action. Neuron, 1997. 19(6): p. 1271-83.

64. Mooslehner, K.A., et al., Mice with very low expression of the vesicular monoamine transporter 2 gene survive into adulthood: potential mouse model for parkinsonism. Mol Cell Biol, 2001. 21(16): p. 5321-31.

65. Caudle, W.M., et al., Reduced vesicular storage of dopamine causes progressive nigrostriatal neurodegeneration. J Neurosci, 2007. 27(30): p. 8138-48. 
946

947

948

949

950

951

952

953

954

955

956

957

958

959

960

961

962

963

964

965

966

967

968

969

970

971

972

973

974

975

976

977

978

979

980

981

982

983

984

985

986

987

988

989

990

991

992

993

994

66. Taylor, T.N., et al., Nonmotor symptoms of Parkinson's disease revealed in an animal model with reduced monoamine storage capacity. J Neurosci, 2009. 29(25): p. 8103-13.

67. Back, S., et al., Neuron-Specific Genome Modification in the Adult Rat Brain Using CRISPR-Cas9 Transgenic Rats. Neuron, 2019.

68. Zimmer, E.R., et al., MicroPET imaging and transgenic models: a blueprint for Alzheimer's disease clinical research. Trends Neurosci, 2014. 37(11): p. 629-41.

69. Andrews-Hanna, J.R., The brain's default network and its adaptive role in internal mentation. Neuroscientist, 2012. 18(3): p. 251-70.

70. Gottlich, M., et al., Altered resting state brain networks in Parkinson's disease. PLoS One, 2013. 8(10): p. e77336.

71. Huang, S., et al., Multisensory Competition Is Modulated by Sensory Pathway Interactions with Fronto-Sensorimotor and Default-Mode Network Regions. J Neurosci, 2015. 35(24): p. 9064-77.

72. Wu, C.W., et al., Synchrony Between Default-Mode and Sensorimotor Networks Facilitates Motor Function in Stroke Rehabilitation: A Pilot fMRI Study. Front Neurosci, 2020. 14: p. 548.

73. Wang, J., et al., Functional reorganization of intra- and internetwork connectivity in major depressive disorder after electroconvulsive therapy. Hum Brain Mapp, 2018. 39(3): p. 1403-1411.

74. Perlbarg, V., et al., Alterations of the nigrostriatal pathway in a 6-OHDA rat model of Parkinson's disease evaluated with multimodal MRI. PLoS One, 2018. 13(9): p. e0202597.

75. Monnot, C., et al., Asymmetric dopaminergic degeneration and levodopa alter functional corticostriatal connectivity bilaterally in experimental parkinsonism. Exp Neurol, 2017. 292: p. 1120.

76. Honey, G.D., et al., Dopaminergic drug effects on physiological connectivity in a human corticostriato-thalamic system. Brain, 2003. 126(Pt 8): p. 1767-81.

77. Kwak, Y., et al., Altered resting state cortico-striatal connectivity in mild to moderate stage Parkinson's disease. Front Syst Neurosci, 2010. 4: p. 143.

78. Costa, R.M., et al., Rapid alterations in corticostriatal ensemble coordination during acute dopamine-dependent motor dysfunction. Neuron, 2006. 52(2): p. 359-69.

79. Gatev, P., O. Darbin, and T. Wichmann, Oscillations in the basal ganglia under normal conditions and in movement disorders. Mov Disord, 2006. 21(10): p. 1566-77.

80. Hammond, C., H. Bergman, and P. Brown, Pathological synchronization in Parkinson's disease: networks, models and treatments. Trends Neurosci, 2007. 30(7): p. 357-64.

81. Eusebio, A., et al., Resonance in subthalamo-cortical circuits in Parkinson's disease. Brain, 2009. 132(Pt 8): p. 2139-50.

82. Brazhnik, E., et al., State-dependent spike and local field synchronization between motor cortex and substantia nigra in hemiparkinsonian rats. J Neurosci, 2012. 32(23): p. 7869-80.

83. Moran, R.J., et al., Alterations in brain connectivity underlying beta oscillations in Parkinsonism. PLoS Comput Biol, 2011. 7(8): p. e1002124.

84. Gerfen, C.R. and D.J. Surmeier, Modulation of striatal projection systems by dopamine. Annu Rev Neurosci, 2011. 34: p. 441-66.

85. Caspers, J., et al., Differential Functional Connectivity Alterations of Two Subdivisions within the Right dIPFC in Parkinson's Disease. Front Hum Neurosci, 2017. 11: p. 288.

86. Owens-Walton, C., et al., Increased functional connectivity of thalamic subdivisions in patients with Parkinson's disease. PLoS One, 2019. 14(9): p. e0222002.

87. Kahn, I., et al., Distinct cortical anatomy linked to subregions of the medial temporal lobe revealed by intrinsic functional connectivity. J Neurophysiol, 2008. 100(1): p. 129-39.

88. Vincent, J.L., et al., Coherent spontaneous activity identifies a hippocampal-parietal memory network. J Neurophysiol, 2006. 96(6): p. 3517-31.

89. Hillary, F.G., et al., Hyperconnectivity is a fundamental response to neurological disruption. Neuropsychology, 2015. 29(1): p. 59-75. 
995

996

997

998

999

1000

1001

1002

1003

1004

1005

1006

1007

1008

1009

1010

1011

1012

1013

1014

1015

1016

1017

1018

1019

1020

1021

1022

1023

1024

1025

1026

1027

1028

1029

1030

1031

1032

1033

1034

1035

1036

1037

1038

1039

1040

1041

1042

1043

90. Klobusiakova, P., et al., Connectivity Between Brain Networks Dynamically Reflects Cognitive Status of Parkinson's Disease: A Longitudinal Study. J Alzheimers Dis, 2019. 67(3): p. 971-984.

91. Mevel, K., et al., The default mode network in healthy aging and Alzheimer's disease. Int J Alzheimers Dis, 2011. 2011: p. 535816.

92. Gorges, M., et al., To rise and to fall: functional connectivity in cognitively normal and cognitively impaired patients with Parkinson's disease. Neurobiol Aging, 2015. 36(4): p. 1727-1735.

93. Helmich, R.C., et al., Spatial remapping of cortico-striatal connectivity in Parkinson's disease. Cereb Cortex, 2010. 20(5): p. 1175-86.

94. O'Gorman Tuura, R.L., C.R. Baumann, and H. Baumann-Vogel, Beyond Dopamine: GABA, Glutamate, and the Axial Symptoms of Parkinson Disease. Front Neurol, 2018. 9: p. 806.

95. Whiting, P.J., The GABAA receptor gene family: new opportunities for drug development. Curr Opin Drug Discov Devel, 2003. 6(5): p. 648-57.

96. Kapogiannis, D., et al., Posteromedial cortex glutamate and GABA predict intrinsic functional connectivity of the default mode network. Neuroimage, 2013. 64: p. 112-9.

97. Douaud, G., et al., Integration of structural and functional magnetic resonance imaging in amyotrophic lateral sclerosis. Brain, 2011. 134(Pt 12): p. 3470-9.

98. Levar, N., et al., Anterior cingulate GABA and glutamate concentrations are associated with resting-state network connectivity. Sci Rep, 2019. 9(1): p. 2116.

99. Lanoue, A.C., G.J. Blatt, and J.J. Soghomonian, Decreased parvalbumin mRNA expression in dorsolateral prefrontal cortex in Parkinson's disease. Brain Res, 2013. 1531: p. 37-47.

100. Lanoue, A.C., et al., Decreased glutamic acid decarboxylase mRNA expression in prefrontal cortex in Parkinson's disease. Exp Neurol, 2010. 226(1): p. 207-17.

101. Ihara, M., et al., Association of vascular parkinsonism with impaired neuronal integrity in the striatum. J Neural Transm (Vienna), 2007. 114(5): p. 577-84.

102. van Nuland, A.J.M., et al., GABAergic changes in the thalamocortical circuit in Parkinson's disease. Hum Brain Mapp, 2020. 41(4): p. 1017-1029.

103. Allen, P., et al., Extrinsic and default mode networks in psychiatric conditions: Relationship to excitatory-inhibitory transmitter balance and early trauma. Neurosci Biobehav Rev, 2019. 99: p. 90-100.

104. Liu, Z., et al., Tissue Specific Expression of Cre in Rat Tyrosine Hydroxylase and Dopamine Active Transporter-Positive Neurons. PLoS One, 2016. 11(2): p. e0149379.

105. Anders Björklund, S.B.D., Dopamine neuron systems in the brain: an update. Trends in Neuroscience, 2007. 30(5): p. 194-202.

106. Ran, F.A., et al., Genome engineering using the CRISPR-Cas9 system. Nat Protoc, 2013. 8(11): p. 2281-2308.

107. Vouillot, L., A. Thelie, and N. Pollet, Comparison of T7E1 and surveyor mismatch cleavage assays to detect mutations triggered by engineered nucleases. G3 (Bethesda), 2015. 5(3): p. 407-15.

108. Huang, Z.R., et al., A Novel Potential Positron Emission Tomography Imaging Agent for Vesicular Monoamine Transporter Type 2. PLoS One, 2016. 11(9): p. e0161295.

109. Ding, Y.S., et al., Pharmacokinetics and in vivo specificity of [11C]dl-threo-methylphenidate for the presynaptic dopaminergic neuron. Synapse, 1994. 18(2): p. 152-60.

110. Wadsworth, H., et al., [(1)(8)F]GE-180: a novel fluorine-18 labelled PET tracer for imaging Translocator protein 18 kDa (TSPO). Bioorg Med Chem Lett, 2012. 22(3): p. 1308-13.

111. Paasonen, J., et al., Functional connectivity under six anesthesia protocols and the awake condition in rat brain. Neuroimage, 2018. 172: p. 9-20.

112. Lopez-Gonzalez, F.J., et al., QModeling: a Multiplatform, Easy-to-Use and Open-Source Toolbox for PET Kinetic Analysis. Neuroinformatics, 2019. 17(1): p. 103-114.

113. Schiffer, W.K., et al., Serial microPET measures of the metabolic reaction to a microdialysis probe implant. J Neurosci Methods, 2006. 155(2): p. 272-84. 
1044 114. Logan, J., Graphical analysis of PET data applied to reversible and irreversible tracers. Nucl Med Biol, 2000. 27(7): p. 661-70.

115. Judenhofer, M.S., et al., Simultaneous PET-MRI: a new approach for functional and morphological imaging. Nat Med, 2008. 14(4): p. 459-65.

1048

1049

116. Ionescu, T., Striatal and prefrontal D2R and SERT distributions contrastingly correlate with defaultmode connectivity. 2021.

1050

1051

1052

117. Matthew Brett, J.-L.A., Romain Valabregue, Jean-Baptiste Poline, Region of interest analysis using an SPM toolbox, in 8th Internation Conference on Functional Mapping of the Human Brain. June 26, 2002: Sendai, Japan.

118. Rubinov, M. and O. Sporns, Complex network measures of brain connectivity: uses and interpretations. Neuroimage, 2010. 52(3): p. 1059-69.

119. Ungerstedt, U. and G.W. Arbuthnott, Quantitative recording of rotational behavior in rats after 6hydroxy-dopamine lesions of the nigrostriatal dopamine system. Brain Res, 1970. 24(3): p. 485-93.

120. West, M.J., Stereological methods for estimating the total number of neurons and synapses: issues of precision and bias. Trends Neurosci, 1999. 22(2): p. 51-61. 


\section{Acknowledgments}

1062

1063

1064

1065

1066

1067

1068

1069

1070

1071

1072

1073

\section{Funding}

$\begin{array}{lll}1075 & - & \text { German Research Foundation to KH } \\ 1076 & \text { - } & \text { Carl-Zeiss Foundation to KH } \\ 1077 & \text { - } & \text { Werner Siemens Foundation to BJP } \\ 1078 & \text { - } & \text { Deutscher Akademischer Austauschdienst to SM, KH }\end{array}$

\section{Author contributions}

$\begin{array}{lll}1080 & \bullet & \text { Conceptualization: KH } \\ 1081 & \bullet & \text { Methodology: SM, TMI } \\ 1082 & \bullet & \text { Software: SM, TMI } \\ 1083 & \bullet & \text { Validation: SM } \\ 1084 & \bullet & \text { Formal analysis: SM, TMI }\end{array}$


1085 Investigation: SM, TMI, AM, RSS, RYC

1086 - Resources: BJP, DK

1087 - Data curation: SM

1088 - Writing - original draft: SM

1089 - Writing - review and editing: SM, TMI, AM, DK, RSS, RYC, BJP, KH

1090 - Supervision: KH

1091 - Project administration: KH

1092 - Funding acquisition: BJP, KH

\section{Competing interests}

1094 The authors declare no conflict of interest.

\section{Data availability}

1096 The original dataset will be made available upon request. 
bioRxiv preprint doi: https://doi.org/10.1101/2021.09.10.459766; this version posted October 11, 2021. The copyright holder for this preprint (which was not certified by peer review) is the author/funder, who has granted bioRxiv a license to display the preprint in perpetuity. It is made available under aCC-BY-NC-ND 4.0 International license.

a

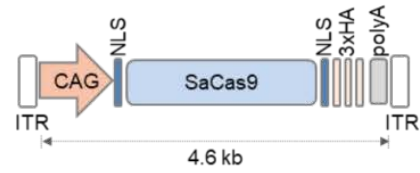

b

C
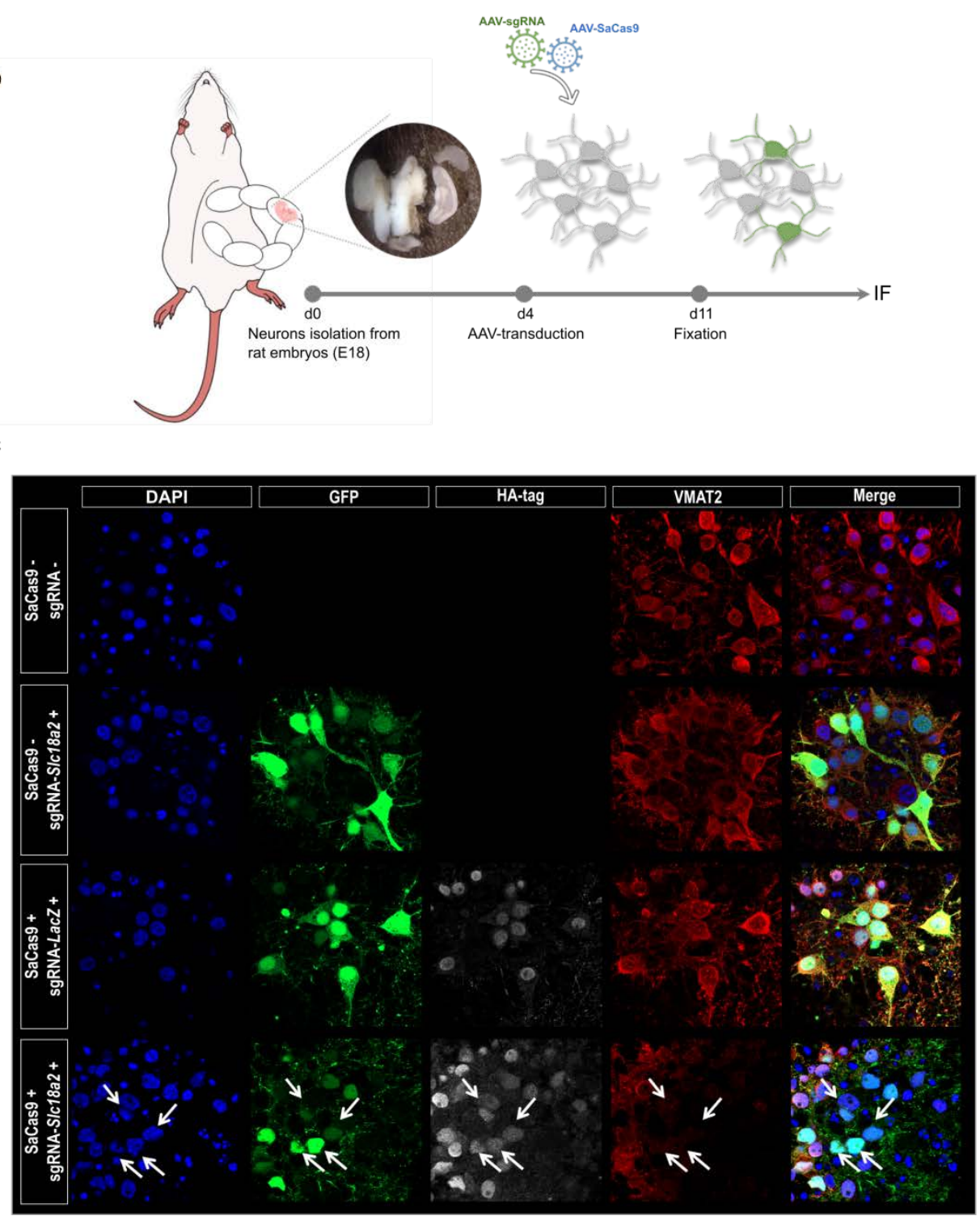

$\operatorname{lacZ}$ (exon 1)

AGGCCCGCACCGATCGCCCTTCCCAACAGTTGCGCAGCCTGAATGGCGAATGGCGCTTTG ...3*

SIc18a2 (exon 2)

5... AGCAGCGCAAGGAACACGATGAACAGGATCAGTTTGCGGGGGTGGCGGCTGTCC...3.
Fig. 1 In vitro validation of CRISPR/SaCas9-induced VMAT2 knockdown in rat primary cortical neurons. (a) AAV-SaCas9 and AAV-sgRNA expression vectors. (b) Experimental design for primary neurons isolation and transduction. (c) VMAT2 immunostaining (red), nuclei labeled with DAPI (blue). GFP 
bioRxiv preprint doi: https://doi.org/10.1101/2021.09.10.459766; this version posted October 11, 2021. The copyright holder for this preprint (which was not certified by peer review) is the author/funder, who has granted bioRxiv a license to display the preprint in perpetuity. It is made available under aCC-BY-NC-ND 4.0 International license.

1104 VMAT2 KD (arrows) is shown in neurons transduced with AAVs carrying SaCas9 and sgRNA-S/c18a2. KD,

1105 knockdown; ITR, inverted terminal repeat; CMV, cytomegalovirus promoter; GFP, green fluorescent protein; CAG, CMV enhancer/chicken $\beta$-actin promoter; NLS, nuclear localization signal; HA-tag, hemagglutinin tag; polyA, polyadenylation signal; polyT, polytermination signal. 
a

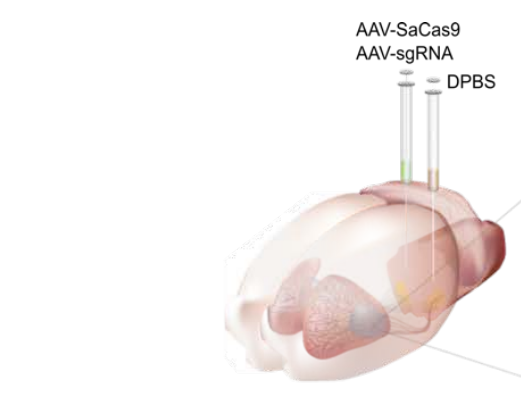

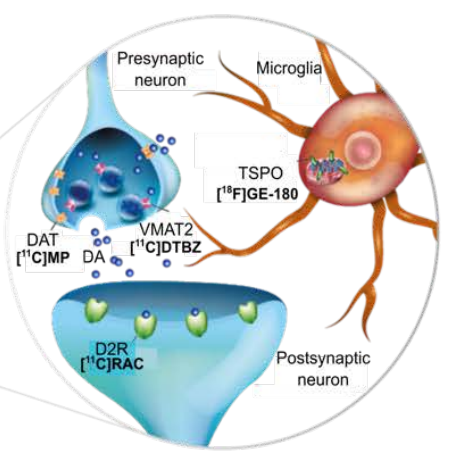

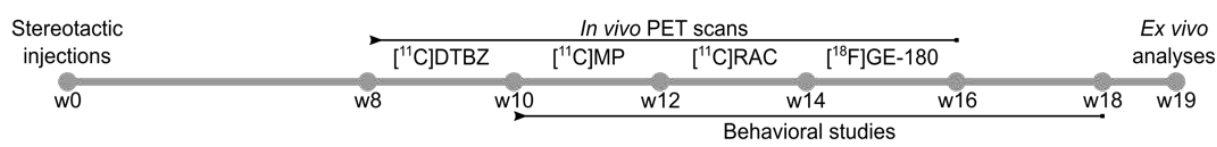

b

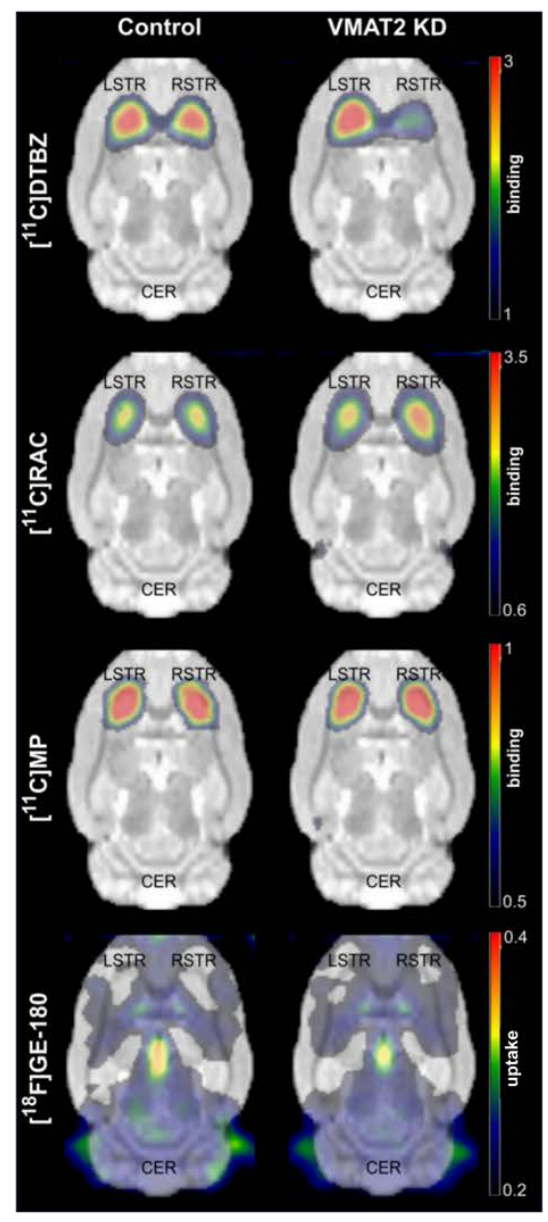

c
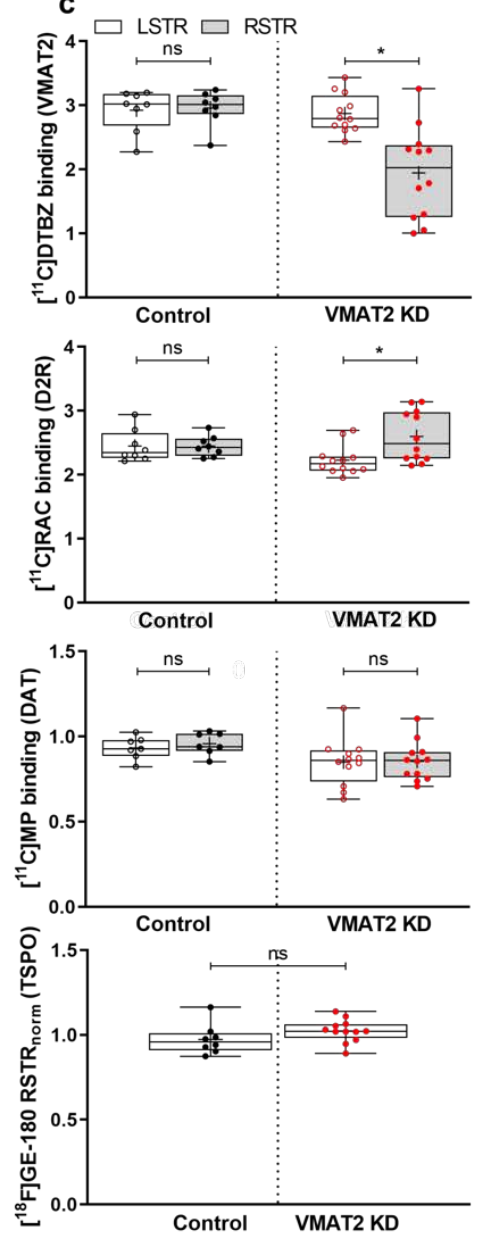

d

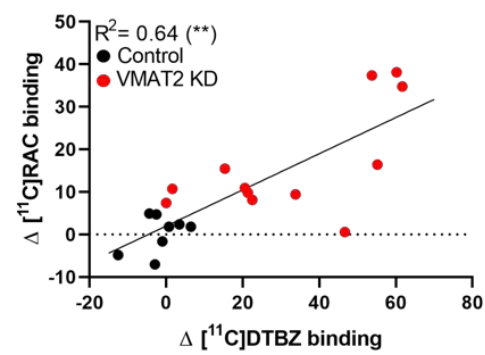

e

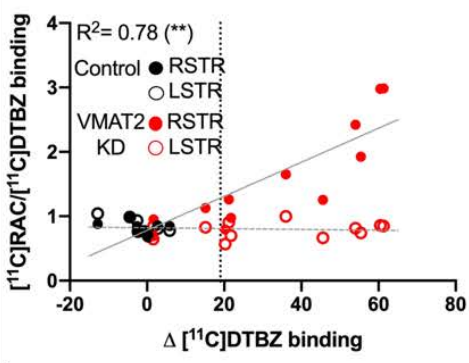

f

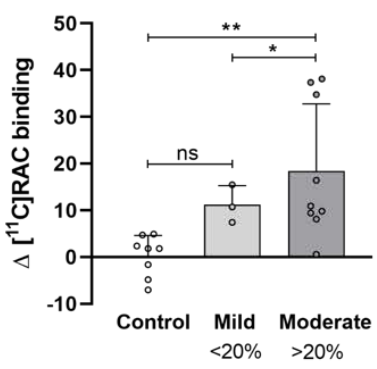

Fig. 2 CRISPR/SaCas9-induced VMAT2 knockdown elicits postsynaptic changes but no nerve terminal loss or neuroinflammation in the adult rat brain. (a) Schematic illustration of the experimental design. (b) Mean binding potential and uptake maps of control and VMAT2 KD rats co-registered to a rat brain atlas. (c) Binding potential values of individual control and VMAT2 KD rats in the left and right striatum. For $\left[{ }^{18} \mathrm{~F}\right] \mathrm{GE}-180$ uptake values normalized to the left striatum are shown. (d) A strong correlation between $\Delta\left[{ }^{11} \mathrm{C}\right] \mathrm{RAC}$ and $\Delta\left[{ }^{11} \mathrm{C}\right] \mathrm{DTBZ}$ binding is shown. (e) Ratio of striatal $\left[{ }^{11} \mathrm{C}\right] \mathrm{RAC}$ and $\left[{ }^{11} \mathrm{C}\right] \mathrm{DTBZ}$ 1116 binding shows prominent $\left[{ }^{11} \mathrm{C}\right] \mathrm{RAC}$ changes when a threshold of $\sim 20 \% \Delta\left[{ }^{11} \mathrm{C}\right] \mathrm{DTBZ}$ binding is reached. 
1117 This threshold was set to separate the VMAT2 KD rats into mild and moderate. (f) Mild and moderate rats could be differentiated based on the postsynaptic changes. ${ }^{*} P<0.01,{ }^{*} P<0.001$, Bonferroni-Sidak corrected. Data are shown as boxplot with the median value (central mark), the mean value (plus sign), interquartile range (boxes edges), and the extreme points of the distribution (whiskers). Control rats $n=8$; VMAT2 KD rats $\mathrm{n}=12$. Mild: $\Delta\left[{ }^{11} \mathrm{C}\right] \mathrm{DTBZ}$ binding < 20\%; Moderate: $\Delta\left[{ }^{11} \mathrm{C}\right] \mathrm{DTBZ}$ binding $\geq 20 \%$. $\left[{ }^{11} \mathrm{C}\right] \mathrm{MP}$, $1122 \quad\left[{ }^{11} \mathrm{C}\right]$ methylphenidate; $\left[{ }^{11} \mathrm{C}\right] \mathrm{RAC}, \quad\left[{ }^{11} \mathrm{C}\right]$ raclopride; LSTR, left striatum; RSTR, right striatum; CER, cerebellum; KD, knockdown; DAT, dopamine transporter; TSPO, translocator protein. 

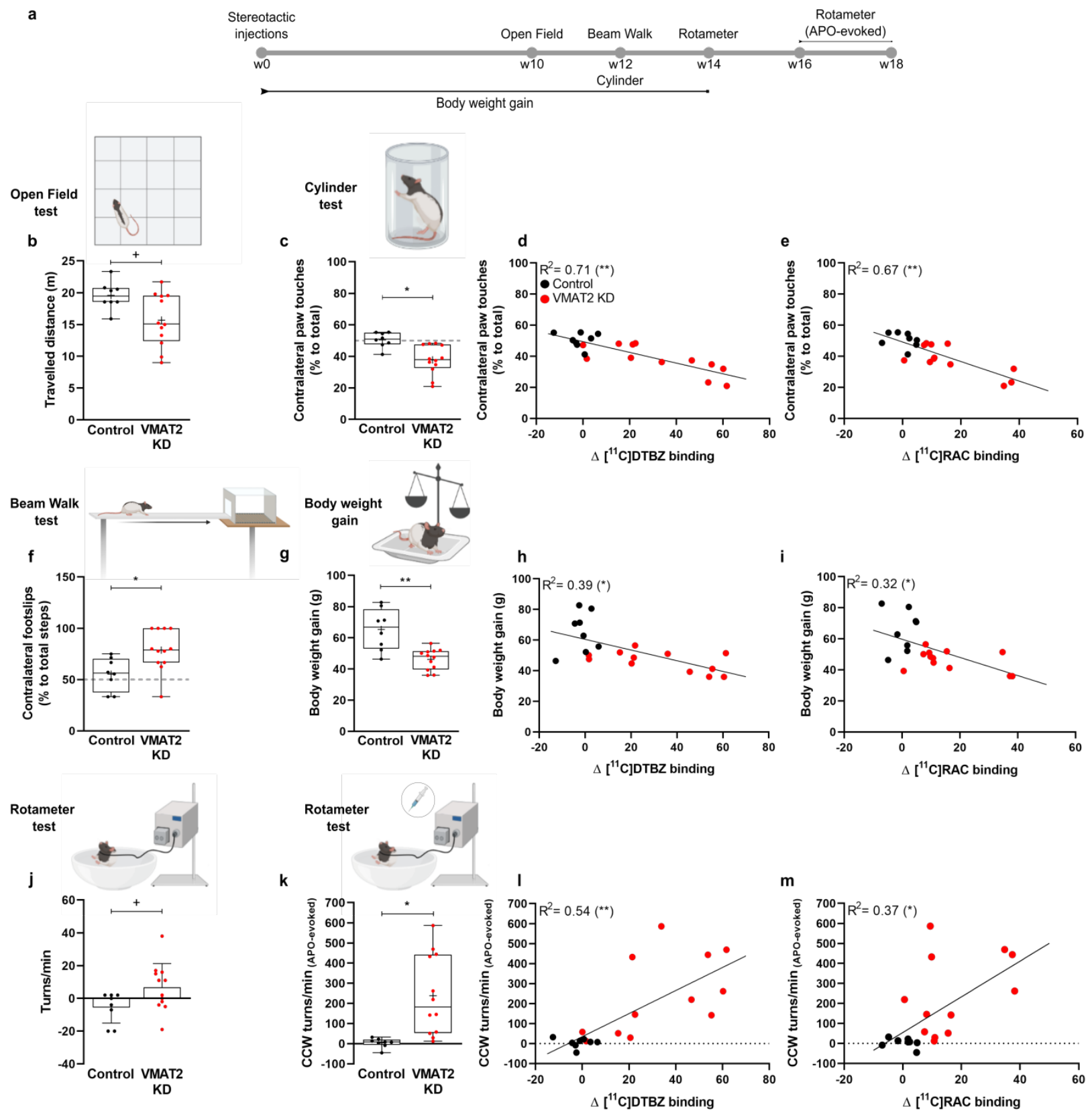

Fig. 3 CRISPR/SaCas9-induced VMAT2 knockdown impairs motor function. (a) Schematic illustration of the behavioral tests. (b) In the open field test, the distance travelled $(\mathrm{m})$ by VMAT2 KD rats was reduced. (c) Cylinder test. VMAT2 KD rats showed a reduction in the contralateral paw touches, compared with controls. Rats performance in the cylinder test strongly correlated with VMAT2 expression changes $\left(\Delta\left[{ }^{11} \mathrm{C}\right] \mathrm{DTBZ}\right.$ binding) and corresponding changes in dopamine availability $\left(\Delta\left[{ }^{11} \mathrm{C}\right] \mathrm{RAC}\right.$ binding) (d,e). (f) In the beam walk test, VMAT2 KD rats displayed a higher number of footslips to the left contralateral side compared with control rats. (g) Body weight assessment 14 weeks after CRISPR/SaCas9 gene-editing showed reduced body weight gain in VMAT2 KD compared with control rats. (h,i) Body weight gain correlated with VMAT2 expression changes $\left(\Delta\left[{ }^{11} \mathrm{C}\right] \mathrm{DTBZ}\right.$ binding) and corresponding changes in dopamine availability ( $\triangle\left[{ }^{11} \mathrm{C}\right] \mathrm{RAC}$ binding). (j) Spontaneous rotation in a novel spherical environment showed increased CW rotations in VMAT2 KD rats compared with control rats. (k) Apomorphine-evoked rotational behavior. VMAT2 KD rats displayed a higher number of CCW rotations compared with control rats in the rotameter test. $(\mathbf{I}, \mathbf{m})$ Apomorphine-evoked rotations exhibited a strong correlation with VMAT2 
1139 expression changes $\left(\Delta\left[{ }^{11} C\right]\right.$ DTBZ binding) and changes in dopamine availability $\left(\Delta\left[{ }^{11} C\right] R A C\right.$ binding). 1140 Data are shown as boxplot with the median value (central mark), the mean value (plus sign), interquartile 1141 range (boxes edges), and the extreme points of the distribution (whiskers). ${ }^{+} P<0.05,{ }^{\star} P<0.01,{ }^{*} P<0.001$. 1142 Control rats $n=8$; VMAT2 KD rats $n=12$. CCW, counter-clockwise; APO, apomorphine; KD, knockdown; $1143\left[{ }^{11} \mathrm{C}\right] \mathrm{RAC},\left[{ }^{11} \mathrm{C}\right]$ raclopride. Illustrations in the figure were created with BioRender.com. 

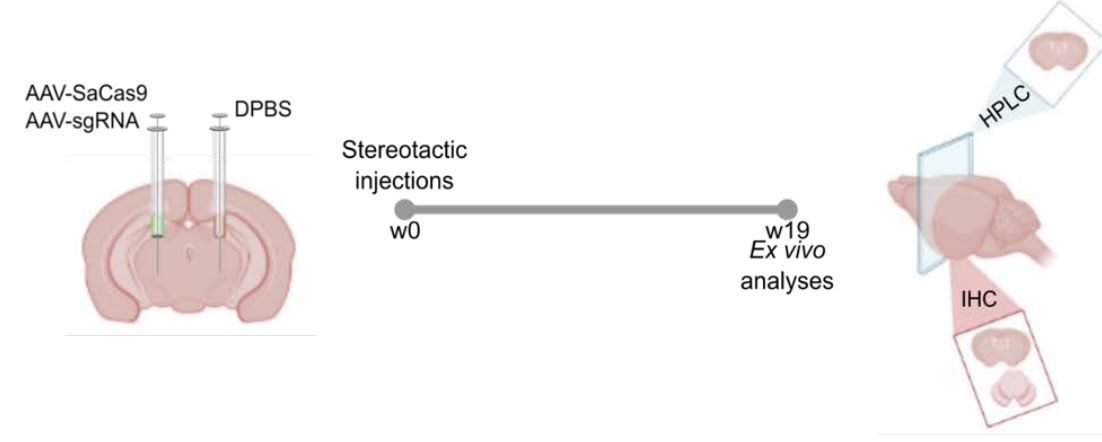

b

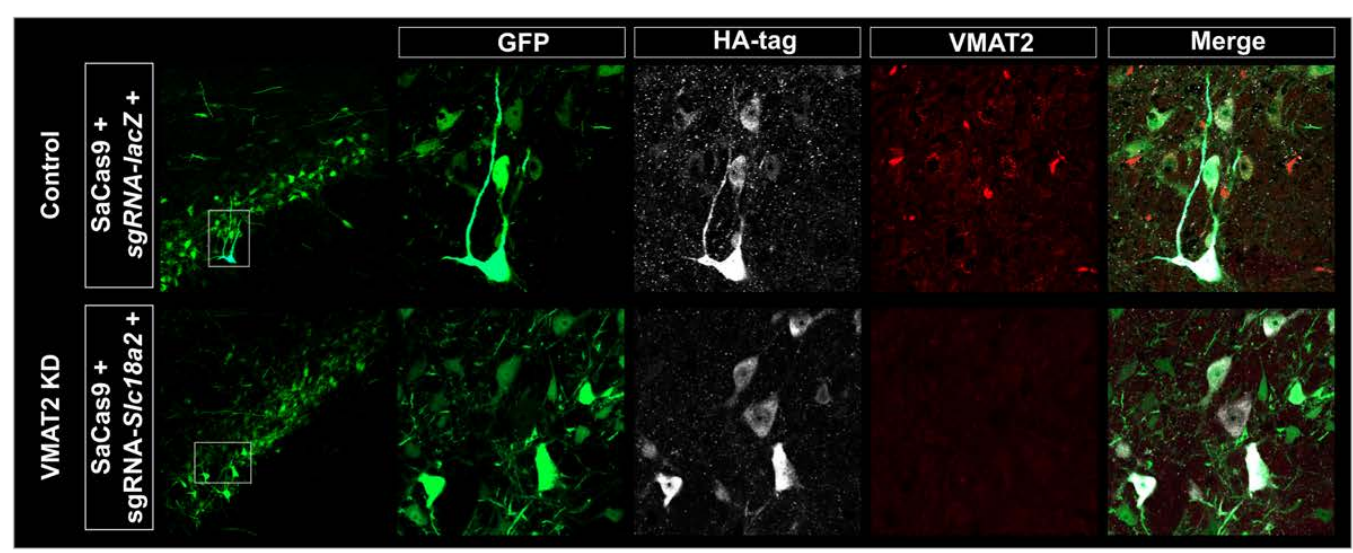

c
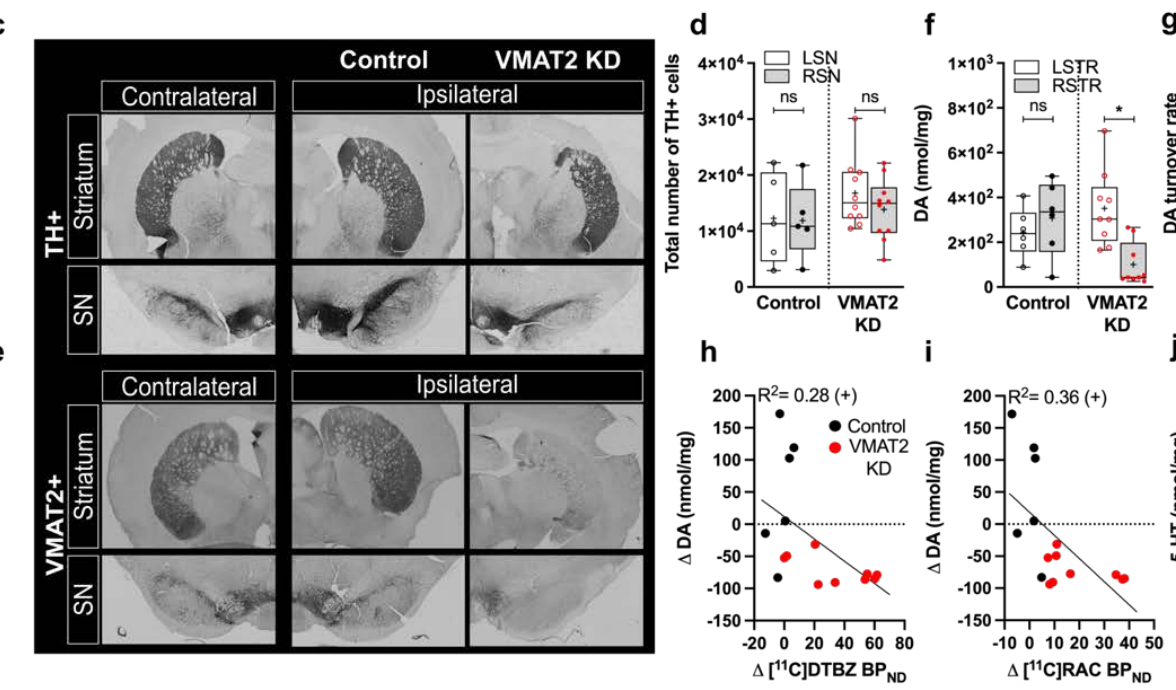

g

Fig. 4 Ex vivo validation of the CRISPR/SaCas9-induced VMAT2 knockdown. (a) Schematic illustration of the ex vivo analyses. (b) Immunofluorescence of nigral sections of control and VMAT2 KD rats confirmed the concomitant expression of SaCas9 and sgRNA. Staining for GFP (AAV-sgRNAs, green), HAtag (AAV-SaCas9, white), and VMAT2 expression (red) for two exemplary rats is shown. VMAT2 expression was largely reduced in the SNc of VMAT2 KD rats. (c,d) TH expression in the ipsilateral and contralateral striatum and SNc of VMAT2 KD $(n=10)$ and control $(n=5)$ rats evidenced no cell loss. (e) VMAT2 immunohistochemistry in the SNc and striatum confirmed large protein reduction in the ipsilateral hemisphere of VMAT2 KD rats. (f) Striatal dopamine, normalized to total protein concentration, was reduced in the ipsilateral striatum of VMAT2 KD $(n=9)$, but not control $(n=6)$ rats. This reduction was paralleled by increased metabolic outcome $(\mathbf{g})$. (h,i) Dopamine changes correlated with the VMAT2 KD extent and postsynaptic changes, deducted from $\left[{ }^{11} \mathrm{C}\right] \mathrm{DTBZ}$ and $\left[{ }^{11} \mathrm{C}\right] \mathrm{RAC}$, respectively. (j) 5 -HT content was unaltered in the striata of control $(n=6)$ and VMAT2 KD $(n=9)$ rats. Data are shown as boxplot with the 
1158 median value (central mark), the mean value (plus sign), interquartile range (boxes edges), and the extreme points of the distribution (whiskers). ${ }^{+} P<0.05,{ }^{*} P<0.01$. GFP, green fluorescent protein; HA-tag, hemagglutinin tag; KD, knockdown; TH, tyrosine hydroxylase; SN, substantia nigra; STR, striatum; $1161 \quad\left[{ }^{11} \mathrm{C}\right] \mathrm{RAC},\left[{ }^{11} \mathrm{C}\right]$ raclopride; DA, dopamine; 5-HT, serotonin. Metabolites' and neurotransmitters' striatal content is reported in Table 3. Illustrations in the figure were created with BioRender.com. 

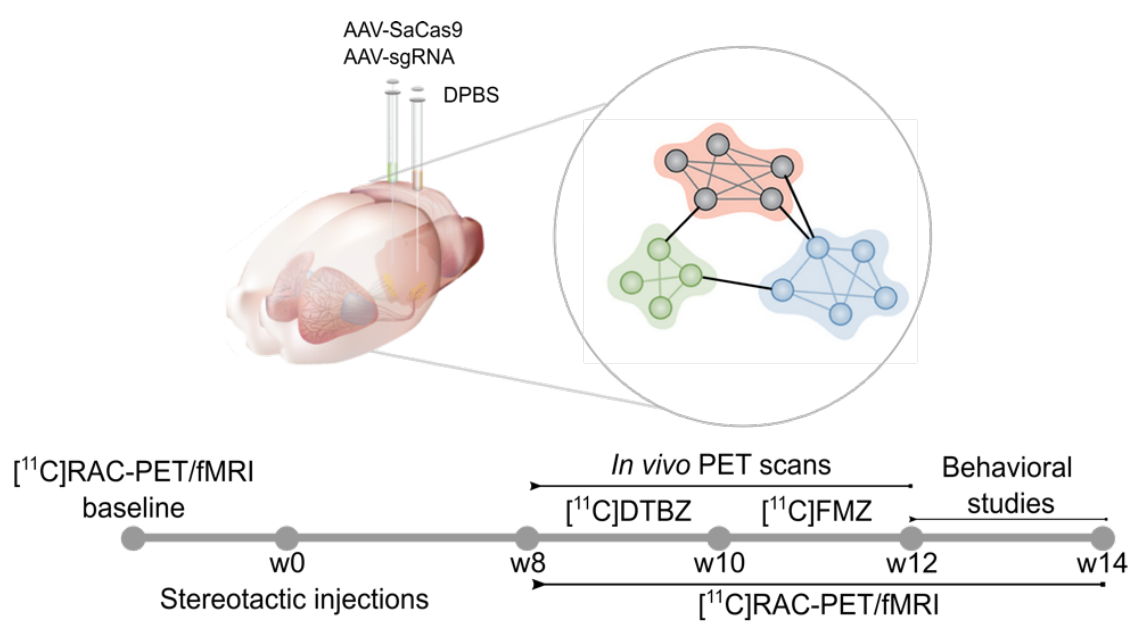

b

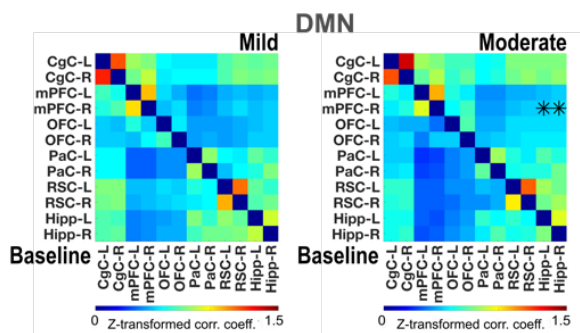

c

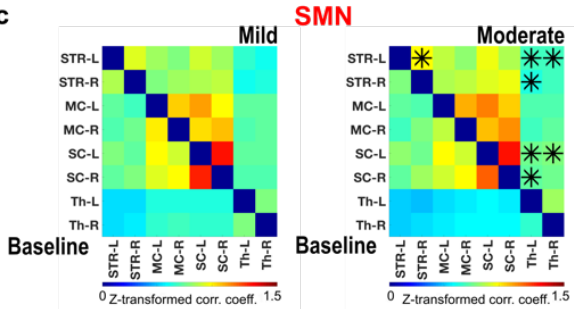

d

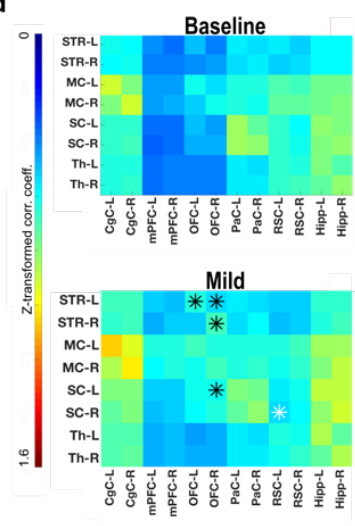

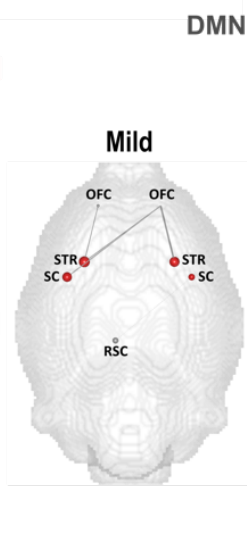

DMN $\leftrightarrow$ SMN

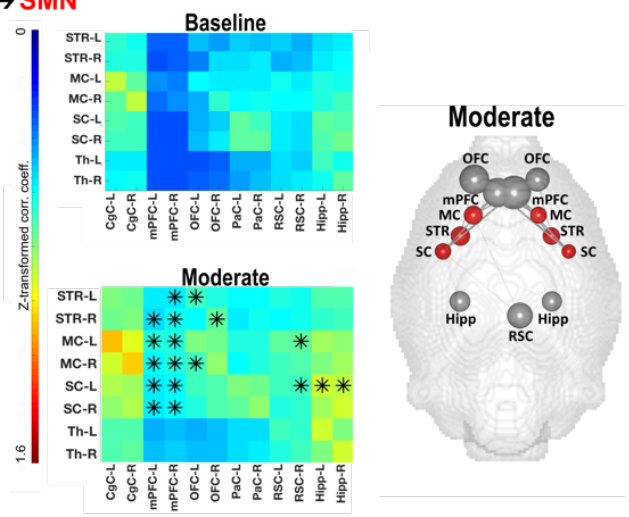

Fig. 5 Increased resting-state functional connectivity after CRISPR/SaCas9-induced VMAT2 knockdown. (a) Schematic illustration of the experimental design. Group level correlation matrices of the DMN (b) and SMN (c) at baseline and after CRISPR/SaCas9-targeting for rats with a mild (left panel) and moderate (right panel) VMAT2 KD. In the moderate KD group rs-FC was increased between the right mPFC and right and left Hipp in the DMN (b) and between the contralateral SC and right and left Th, as well as between the left STR and right and left Th, in the SMN (c). (d) Internetwork rs-FC changes in the mild KD group indicated increased rs-FC between anterior regions of the DMN and the SMN (between the left OFC and STR, and between the right OFC and the left SC, and the right and left STR). Conversely, rsFC was decreased between regions of the posterior DMN and the SMN (between the left RSC and right $\mathrm{SC}$ ). In the moderate KD group, DMN-SMN rs-FC was increased. Brain graphs, right to the matrices, illustrate the nodes and edges (raw values) that demonstrated $\mathrm{rs}-\mathrm{FC}$ changes to baseline (\%). ${ }^{*} P<0.01$. Mild: $\Delta\left[{ }^{11}\right.$ C]DTBZ binding <20\%, $\mathrm{n}=13$; Moderate: $\Delta\left[{ }^{11} \mathrm{C}\right] \mathrm{DTBZ}$ binding $\geq 20 \%, \mathrm{n}=10$. $\mathrm{KD}$, knockdown; DMN, default-mode network; SMN, sensorimotor network. mPFC, medial prefrontal cortex; Hipp, hippocampus; SC, somatosensory cortex; Th, thalamus; STR, striatum; OFC, orbitofrontal cortex; RSC, retrosplenial cortex. Abbreviations of brain regions considered for the analysis of the fMRI data, including their respective volumes, are reported in Table 2. 


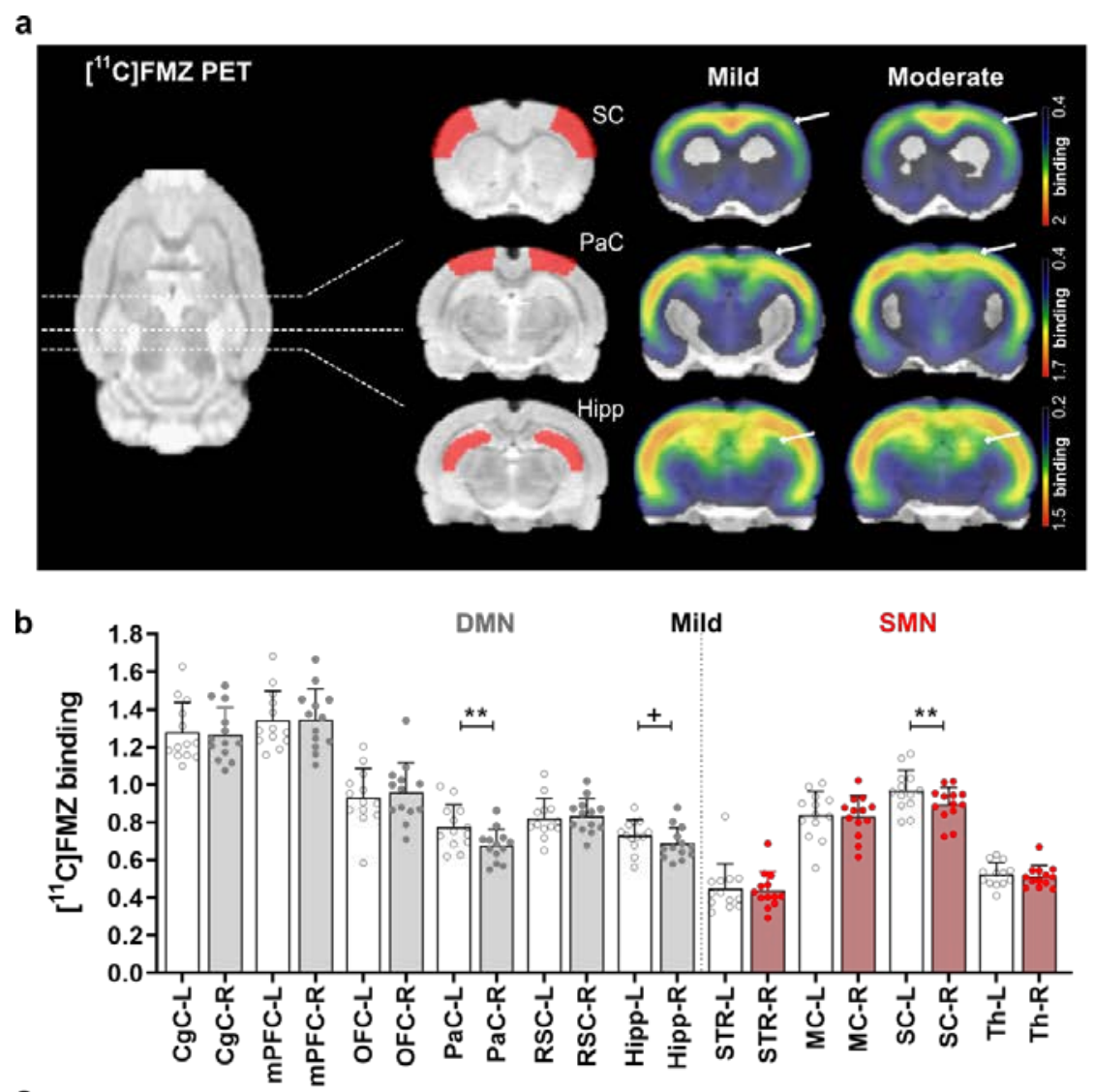

c

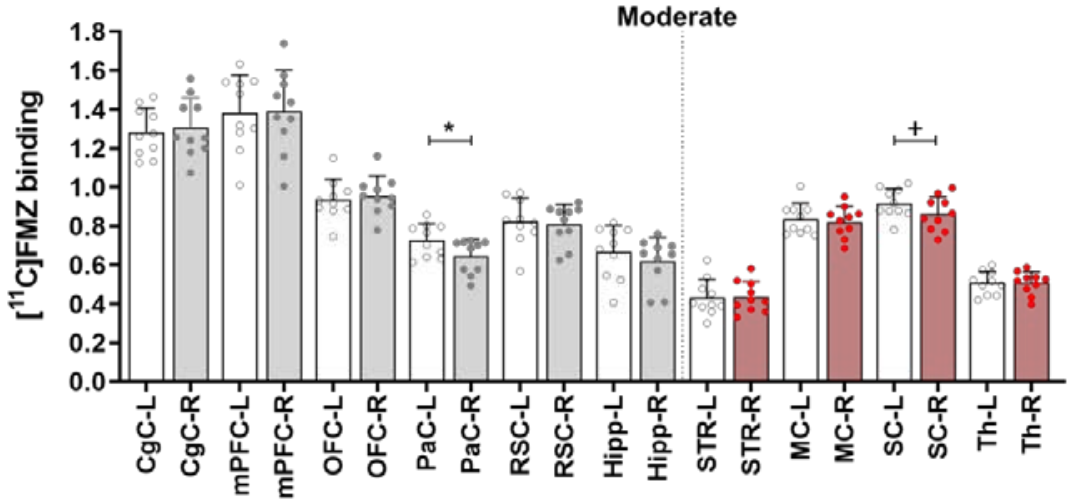

Fig. 6 CRISPR/SaCas9-induced VMAT2 knockdown alters GABA signaling. (a) $\left[{ }^{11} \mathrm{C}\right] \mathrm{FMZ}$ mean binding potential maps of mild and moderate VMAT2 KD rats co-registered to a rat brain atlas. Arrows and ROls in coronal sections indicate brain regions of the DMN and SMN with altered $\left[{ }^{11} \mathrm{C}\right] \mathrm{FMZ}$ binding. $\left[{ }^{11} \mathrm{C}\right] \mathrm{FMZ}$ binding potentials from VOI-based analysis in DMN and SMN regions of rats with mild (b) and moderate (c) VMAT2 KD. $\left[{ }^{11} \mathrm{C}\right] \mathrm{FMZ}$ binding was decreased in the right $\mathrm{PaC}, \mathrm{SC}$, and Hipp of mild KD rats (b) and right $\mathrm{PaC}$ and SC of moderate KD rats (c). ${ }^{+} P<0.05,{ }^{*} P<0.01,{ }^{*} P<0.001$, Bonferroni-Sidak corrected. Data are shown as mean \pm SD. Mild: $\Delta\left[{ }^{11} \mathrm{C}\right] \mathrm{DTBZ}$ binding <20\%, $\mathrm{n}=13$; Moderate: $\Delta\left[{ }^{11} \mathrm{C}\right] \mathrm{DTBZ}$ binding $\geq 20 \%$, $\mathrm{n}=10$. KD, knockdown; $\left[{ }^{11} \mathrm{C}\right] \mathrm{FMZ},\left[{ }^{11} \mathrm{C}\right]$ flumazenil; DMN, default-mode network; SMN, sensorimotor network. PaC, parietal cortex; Hipp, hippocampus; SC; somatosensory cortex. Abbreviations of brain 1192 regions, including their respective volumes, are reported in Table 2. 


\section{Supplementary Figures}

\section{$\%$ editing}

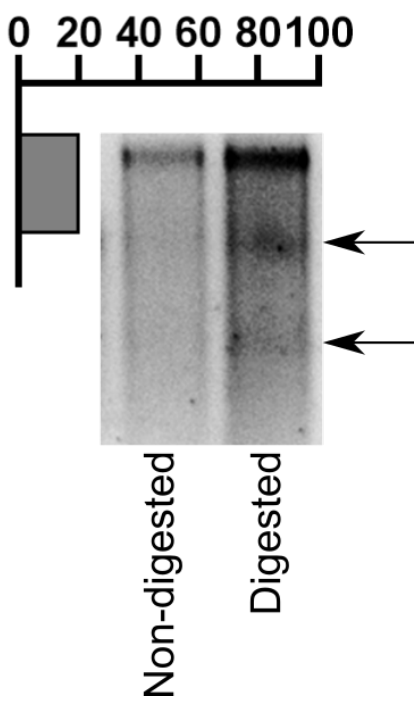

1195 Supplementary Fig. 1 In vitro validation of CRISPR/SaCas9-induced VMAT2 knockdown in rat primary cortical neurons. One-week post-transduction with conditional vectors for SaCas9 and sgRNASlc18a2, neurons were processed for the Surveyor assay. The expected cleavage products (arrows), and estimated editing of $20 \%$, could be seen for the digested DNA. 
a

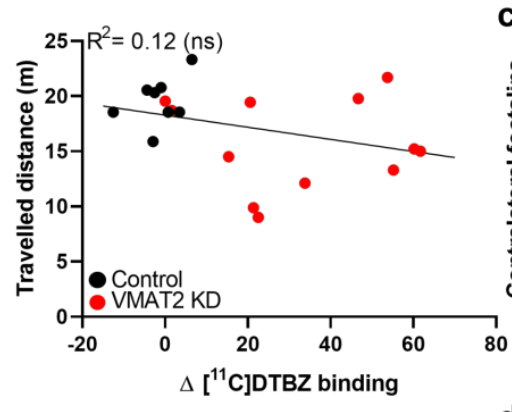

b

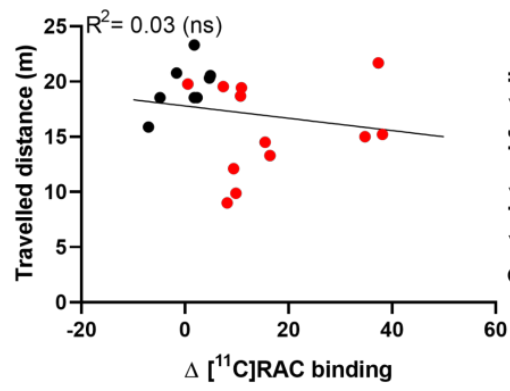

c

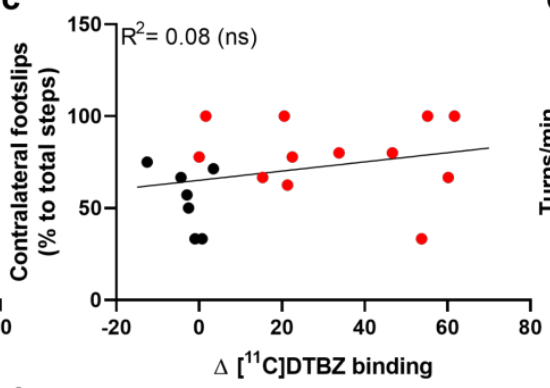

d

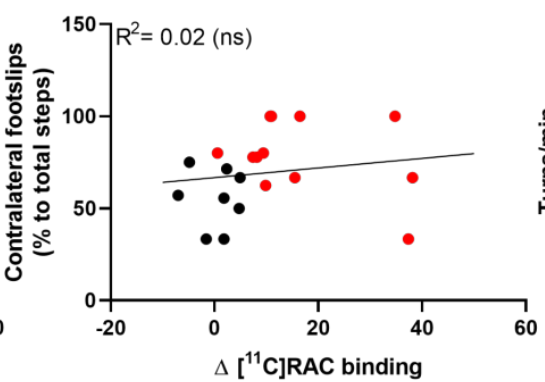

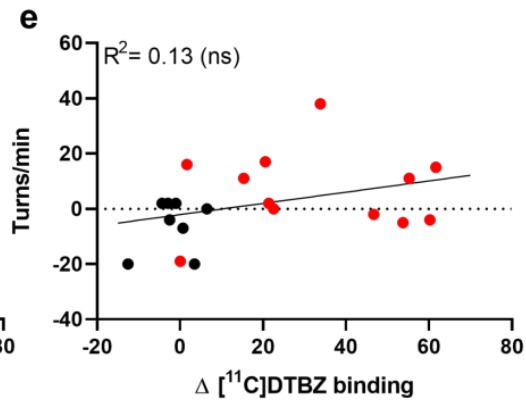

f

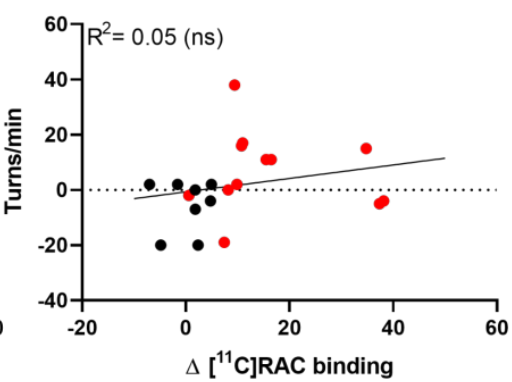

Supplementary Fig. 2 CRISPR/SaCas9-induced VMAT2 knockdown, postsynaptic changes, and motor impairment do not correlate for whole-body movements. No correlation was observed between locomotor activity and (a) VMAT2 expression changes $\left(\Delta\left[{ }^{11} \mathrm{C}\right] \mathrm{DTBZ}\right.$ binding), or (b) dopamine availability $\left(\Delta\left[{ }^{11} \mathrm{C}\right] \mathrm{RAC}\right.$ binding). The number of contralateral footslips in the beam walk test did not correlate with (c) changes in VMAT2 expression ( $\Delta\left[{ }^{11} \mathrm{C}\right] \mathrm{DTBZ}$ binding) or (d) dopamine availability $\left(\Delta\left[{ }^{11} \mathrm{C}\right] \mathrm{RAC}\right.$ binding). Spontaneous rotations did not correlate with (e) changes in VMAT2 expression $\left(\Delta\left[{ }^{11} C\right] D T B Z\right.$ binding $)$ or $(f)$ dopamine availability $\left(\Delta\left[{ }^{11} C\right] R A C\right.$ binding). ns $P \geq 0.05$. Control rats $n=8$; VMAT2 KD rats $n=12$. KD, knockdown; $\left[{ }^{11} \mathrm{C}\right] \mathrm{RAC},\left[{ }^{11} \mathrm{C}\right]$ raclopride. 
a

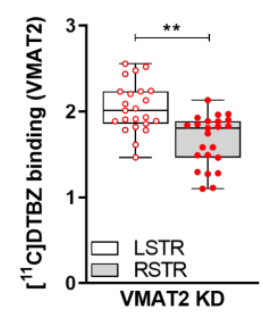

C

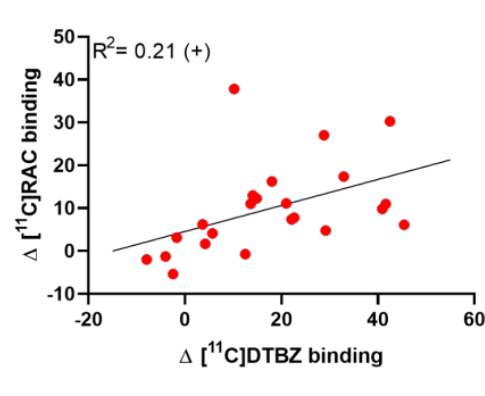

e

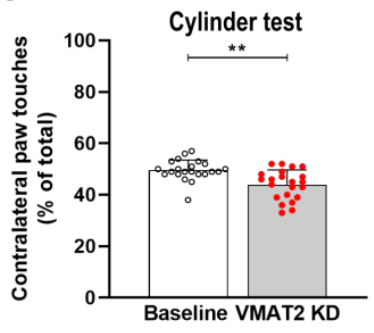

b

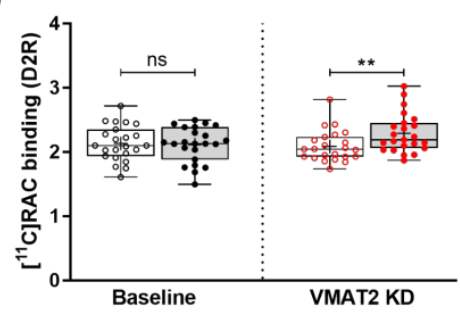

d

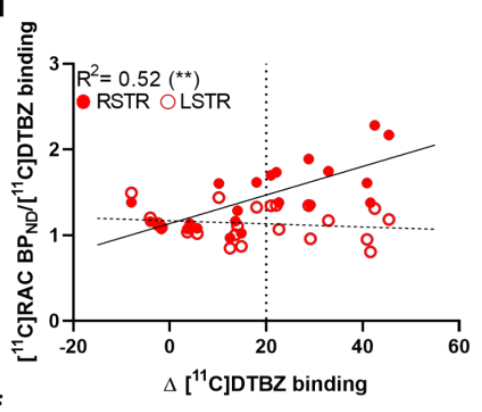

f

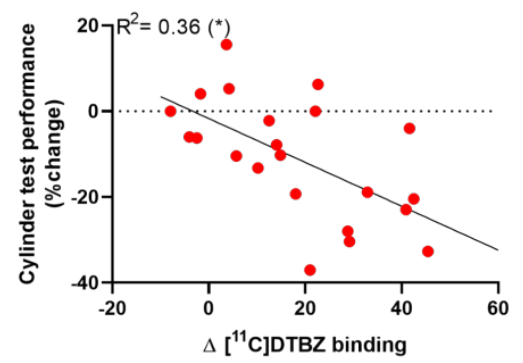

$\mathbf{g}$

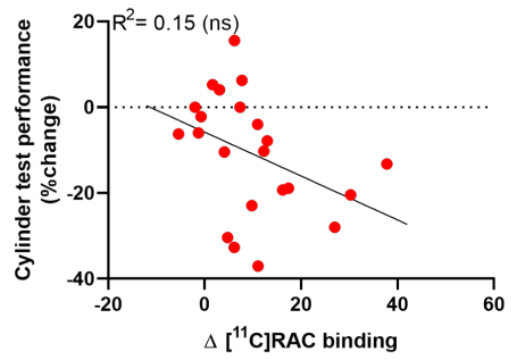

1210

1211

1212

1213

1214

1215

1216

1217

1218

1219

1220

1221

1222

1223

Supplementary Fig. 3 Reproducibility of the CRISPR/SaCas9-induced VMAT2 knockdown. (a) $\left[{ }^{11} \mathrm{C}\right] \mathrm{DTBZ}$ in VMAT2 $\mathrm{KD}$ rats and (b) $\left[{ }^{11} \mathrm{C}\right] \mathrm{RAC}$ PET at baseline and after CRISPR/SaCas9-induced VMAT2 KD. (c) A strong correlation between $\Delta\left[{ }^{11} \mathrm{C}\right] \mathrm{RAC}$ and $\Delta\left[{ }^{11} \mathrm{C}\right] \mathrm{DTBZ}$ binding is shown. (d) At $20 \%$ VMAT2 KD ( $\Delta\left[{ }^{11} C\right] D T B Z$ binding), D2R binding ( $\Delta\left[{ }^{11} C\right] R A C$ binding) prominently increased. This threshold was therefore set to separate the rats into mild and moderate. Data are shown as boxplot with the median value (central mark), the mean value (plus sign), interquartile range (boxes edges), and the extreme points of the distribution (whiskers). Baseline $n=23$, VMAT2 KD $n=23$. (e) Cylinder test at baseline $(n=22)$ and 12 - 14 weeks after CRISPR/SaCas9 gene-editing $(n=22)$. VMAT2 KD rats showed reduced contralateral paw touches compared to baseline. Rats performance in the cylinder test, \% change from baseline, correlated with reduced VMAT2 expression $\left(\Delta\left[{ }^{11} \mathrm{C}\right] \mathrm{DTBZ}\right.$ binding) $(\mathrm{f})$, but not with dopamine availability $\left(\Delta\left[{ }^{11} \mathrm{C}\right] \mathrm{RAC}\right.$ binding) (g). Data are shown as mean $\pm \mathrm{SD} .{ }^{+} P<0.05,{ }^{\star} P<0.01,{ }^{\star \star} P<0.001 . \mathrm{KD}$, knockdown; $\left[{ }^{11} \mathrm{C}\right] \mathrm{RAC},\left[{ }^{11} \mathrm{C}\right]$ raclopride. 
a

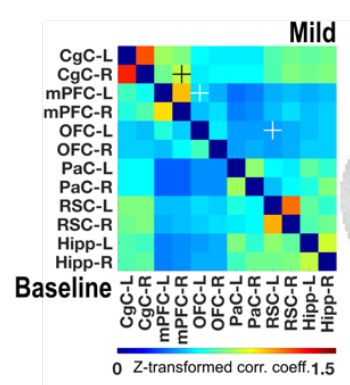

b

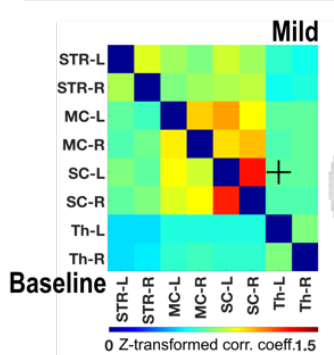

C

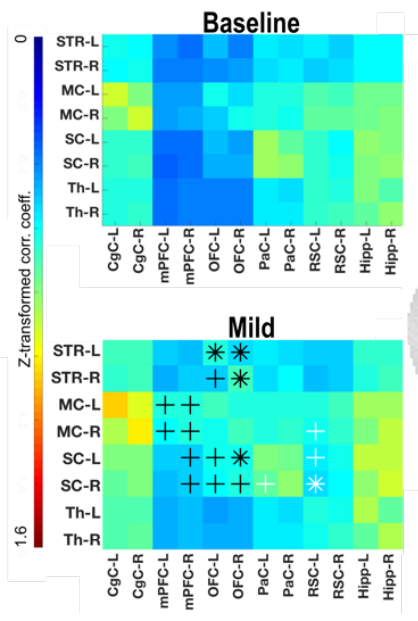

DMN

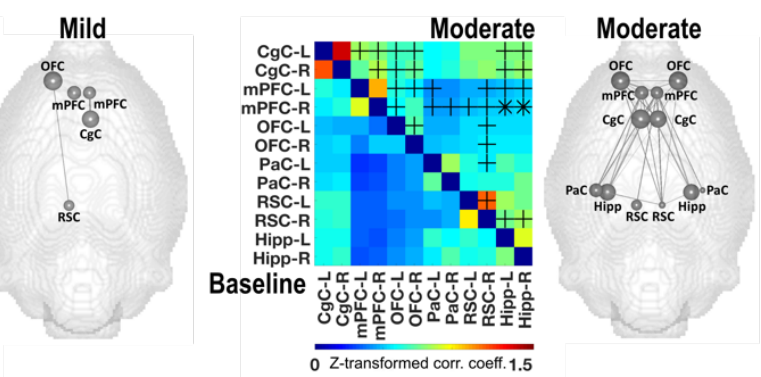

SMN

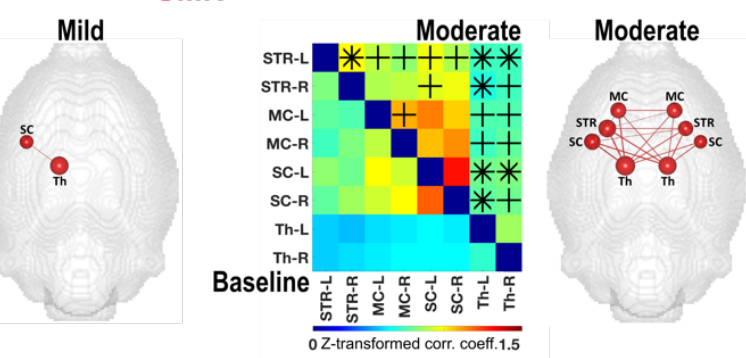

DMN $\leftrightarrow$ SMN
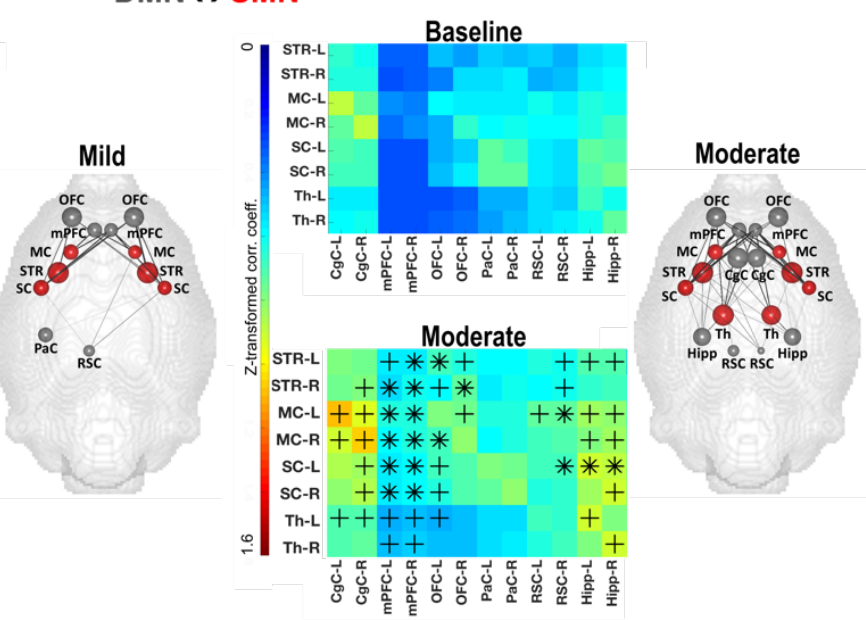

Supplementary Fig. 4 Increased resting-state functional connectivity after CRISPR/SaCas9-induced VMAT2 knockdown. Group level correlation matrices of the DMN (a) and SMN (b) at baseline and after VMAT2 KD for mild (left panel) and moderate (right panel) rats. Brain graphs, right to the matrices, illustrate the nodes and edges (raw values) that demonstrated rs-FC changes to baseline (\%) in DMN (a) and SMN (b). (a) In rats with mild VMAT2 KD, we observed divergent rs-FC changes to baseline in regions of the anterior DMN (left mPFC and OFC, right MPFC and $\mathrm{CgC}$ ). In rats with moderate VMAT2 KD, we observed rs-FC increase in most regions of the anterior DMN, extending to the posterior DMN (RSC bilaterally, right RSC, and Hipp bilaterally). (b) In rats with mild VMAT2 KD, we observed rs-FC increase between the contralateral SC and Th, which intensified in moderate VMAT2 KD rats and extended to most of the regions of the SMN. (c) Group level correlation matrices of the DMN-SMN rs-FC changes at baseline and after VMAT2 KD for mild (left panel) and moderate (right panel) rats. Brain graphs, right to the matrices, illustrate the nodes and edges (raw values) that demonstrated internetwork rs-FC changes to baseline (\%). Internetwork FC changes in rats with mild VMAT2 KD indicated increased rs-FC between anterior regions of the DMN and the SMN (between OFC and STR, SC bilaterally, between right MPFC and MC, SC bilaterally), and decreased rs-FC between posterior regions of the DMN and the SMN (between left RSC and right MC, and SC bilaterally). Increased DMN-SMN rs-FC was found in rats with moderate VMAT2 KD. 
1241 Internetwork rs-FC alterations extended between CgC/Hipp, and SC, MC, Th. ${ }^{+} P<0.05$, ${ }^{*} P<0.01$. Mild: $1242 \Delta\left[{ }^{11}\right.$ C]DTBZ binding $<20 \%, \mathrm{n}=13$; Moderate: $\Delta\left[{ }^{11} \mathrm{C}\right] \mathrm{DTBZ}$ binding $\geq 20 \%, \mathrm{n}=10 . \mathrm{KD}$, knockdown; DMN, 1243 default-mode network; SMN, sensorimotor network. mPFC, medial prefrontal cortex; OFC, orbitofrontal 1244 cortex; CgC, cingulate cortex; RSC, retrosplenial cortex; Hipp, hippocampus; SC, somatosensory cortex; 1245 Th, thalamus; STR, striatum; MC, motor cortex. Abbreviations of brain regions considered for the analysis 1246 of the fMRI data, including their respective volumes, are reported in Table 2. 


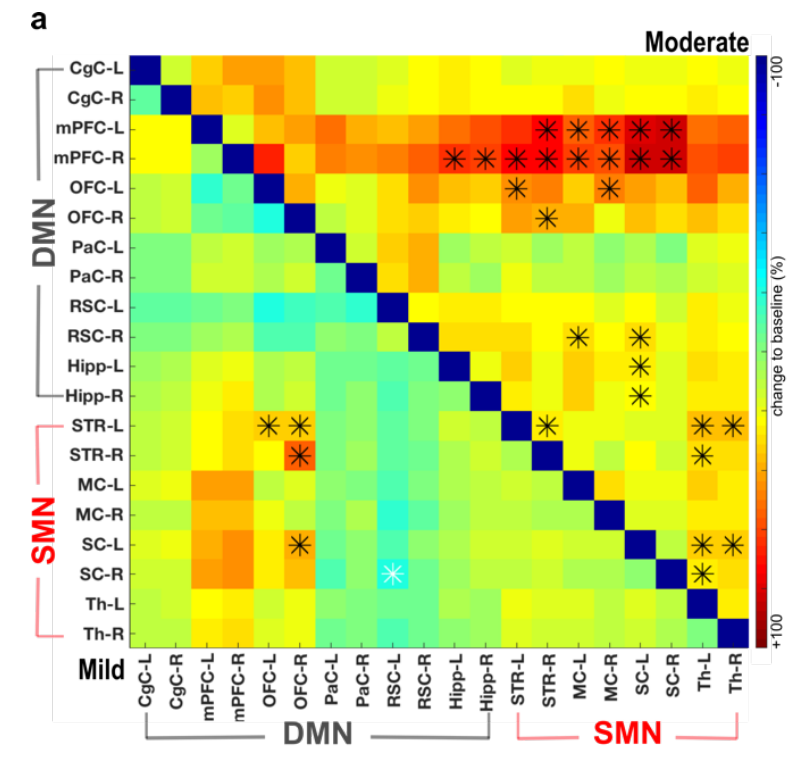

b

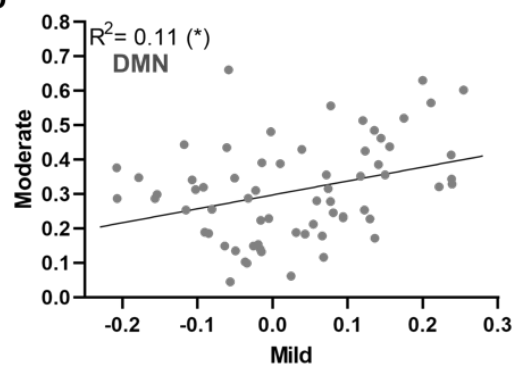

e

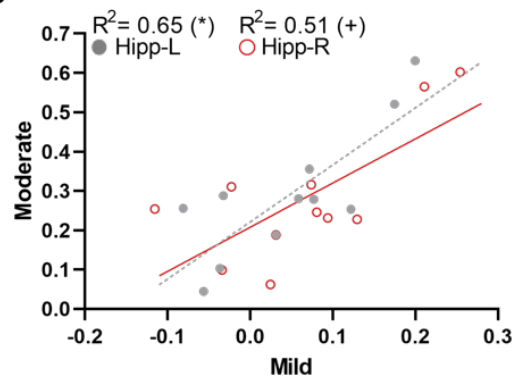

C

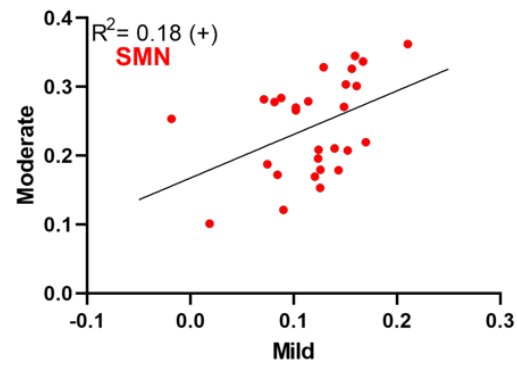

f

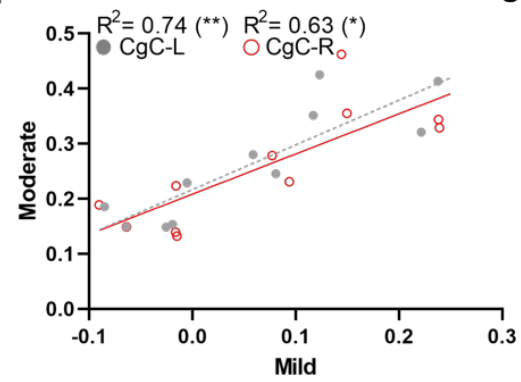

g d
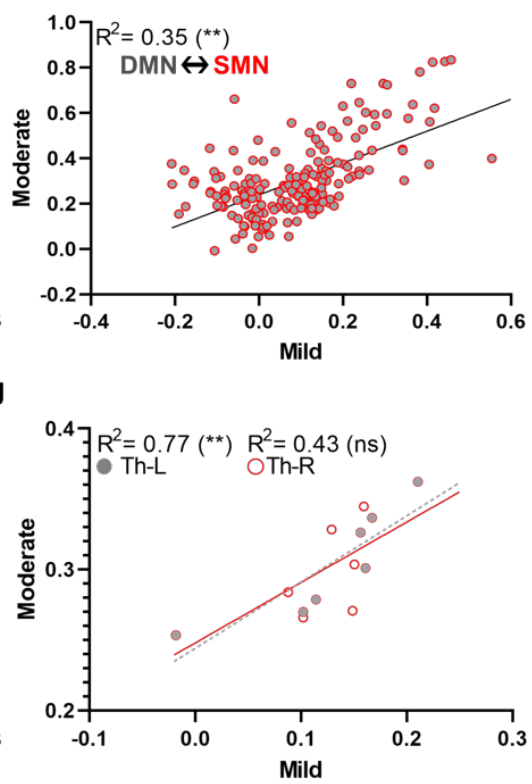

Supplementary Fig. 5 Node correlation analyses evidence pattern similarity and a linear relationship between the magnitude of the resting-state functional connectivity changes in mild and moderate rats. (a) Group level correlation matrices of the intraregional and internetwork rs-FC changes to baseline (\%) for mild and moderate VMAT2 KD rats. (b-d) Group level intraregional and internetwork rs-FC changes correlated linearly between the two groups. Node correlation analysis between mild and moderate VMAT2 KD rats indicated a linear increase in the magnitude of the rs-FC changes to baseline in the right and left Hipp (e), and CgC (f). (g) Rs-FC changes (\%) in the SMN correlated linearly between mild and moderate VMAT2 KD rats in the left, but not in the right Th. Our data suggest a similarity in the pattern of the intraregional and internetwork rs-FC changes between rats with mild and moderate VMAT2 KD and a linear relationship between the magnitude of the rs-FC changes. ${ }^{+} P<0.05,{ }^{*} P<0.01,{ }^{*} P<0.001$. Mild: $\Delta\left[{ }^{11}\right.$ C]DTBZ binding <20\%, $\mathrm{n}=13$; Moderate: $\Delta\left[{ }^{11} \mathrm{C}\right] \mathrm{DTBZ}$ binding $\geq 20 \%, \mathrm{n}=10 . \mathrm{KD}$, knockdown; DMN, default-mode network; SMN, sensorimotor network. Hipp, hippocampus; CgC, cingulate cortex. Th, thalamus. Abbreviations of brain regions considered for the analysis of the fMRI data, including their respective volumes, are reported in Table 2. 

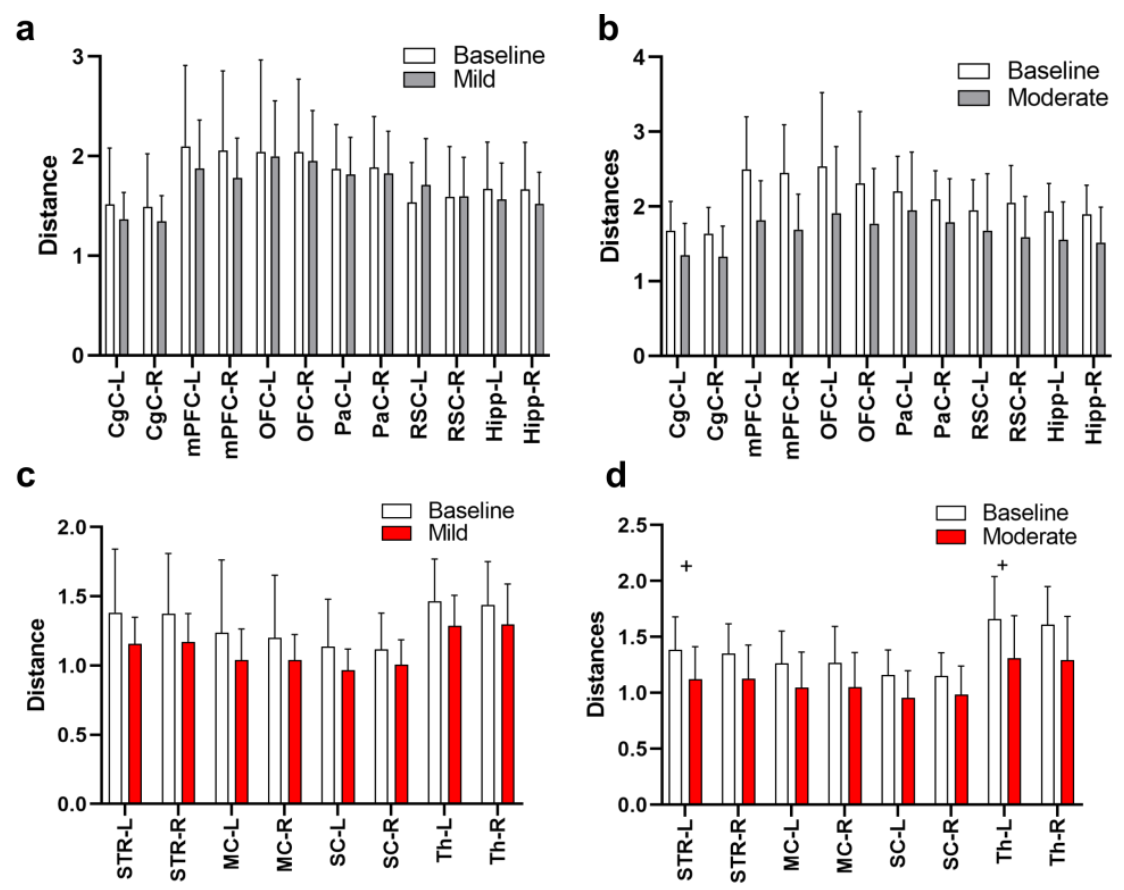

Supplementary Fig. 6 Graph theoretical analyses reveal lateralized recruitment of SMN regions following the CRISPR/SaCas9-induced VMAT2 moderate knockdown. Quantitative graph theoretical analyses of the global mean connection distance indicated no changes for mild (a) and moderate (b) VMAT2 KD rats in the DMN. (c) Rats with mild VMAT2 KD displayed no changes in the SMN. (d) Instead, rats with moderate VMAT2 KD presented shorter functional paths to the nodes in the contralateral Th and STR. Data are shown as mean $\pm \mathrm{SD}$. ${ }^{+} P<0.05$, Bonferroni-Sidak-corrected. Mild: $\Delta\left[{ }^{11} \mathrm{C}\right] \mathrm{DTBZ}$ binding $<20 \%, n=13$; Moderate: $\Delta\left[{ }^{11}\right.$ C]DTBZ binding $\geq 20 \%, n=10$. KD, knockdown; DMN: default-mode network, SMN: sensorimotor network; Th: Thalamus; STR: striatum. Abbreviations of brain regions considered for the analysis of the fMRI data, including their respective volumes, are reported in Table 2. 
bioRxiv preprint doi: https://doi.org/10.1101/2021.09.10.459766; this version posted October 11, 2021. The copyright holder for this preprint (which was not certified by peer review) is the author/funder, who has granted bioRxiv a license to display the preprint in perpetuity. It is made available under aCC-BY-NC-ND 4.0 International license.

\section{Tables}

1275 Table 1: sgRNAs sequences.

1276

1277

1278

\begin{tabular}{|c|c|c|}
\hline SgRNAs & $\begin{array}{c}\text { DNA Target Sequences } \\
\text { ''- 3' }\end{array}$ & $\begin{array}{c}\text { PAM (NNGRRT) } \\
\mathbf{5}^{\prime}-\mathbf{3}^{\prime}\end{array}$ \\
\hline S/c18a2 & CGATGAACAGGATCAGTTTGC & GCGAGT \\
\hline lacZ & CCTTCCCAACAGTTGCGCAGC & CTGAAT \\
\hline
\end{tabular}

1279

1280

1281

1282

1283

1284

1285

1286

1287

1288 
Table 2: Brain regions included in the Paxinos rat brain atlas, including their respective volumes and abbreviations.

\begin{tabular}{|c|c|c|c|}
\hline Brain region (ROI) & Hemisphere & $\begin{array}{l}\text { ROI volume } \\
{\left[\mathrm{mm}^{3}\right]}\end{array}$ & Abbreviation \\
\hline \multirow{2}{*}{ Striatum } & left & \multirow{2}{*}{43.552} & \multirow{2}{*}{ STR } \\
\hline & right & & \\
\hline \multirow{2}{*}{ Cingulate Cortex } & left & \multirow{2}{*}{14.480} & \multirow{2}{*}{$\mathrm{CgC}$} \\
\hline & right & & \\
\hline \multirow{2}{*}{$\begin{array}{l}\text { Medial Prefrontal } \\
\text { Cortex }\end{array}$} & left & \multirow{2}{*}{6.304} & \multirow{2}{*}{ mPFC } \\
\hline & right & & \\
\hline \multirow{2}{*}{ Motor Cortex } & left & \multirow{2}{*}{32.608} & \multirow{2}{*}{ MC } \\
\hline & right & & \\
\hline \multirow{2}{*}{ Orbitofrontal Cortex } & left & \multirow{2}{*}{18.936} & \multirow{2}{*}{ OFC } \\
\hline & right & & \\
\hline \multirow{2}{*}{ Parietal Cortex } & left & \multirow{2}{*}{7.632} & \multirow{2}{*}{$\mathrm{PaC}$} \\
\hline & right & & \\
\hline \multirow{2}{*}{ Retrosplenial Cortex } & left & \multirow{2}{*}{18.920} & \multirow{2}{*}{ RSC } \\
\hline & right & & \\
\hline \multirow{2}{*}{ Somatosensory Cortex } & left & \multirow{2}{*}{71.600} & \multirow{2}{*}{ SC } \\
\hline & right & & \\
\hline \multirow{2}{*}{ Hippocampus } & left & \multirow{2}{*}{25.064} & \multirow{2}{*}{ Hipp } \\
\hline & right & & \\
\hline \multirow{2}{*}{ Thalamus } & left & \multirow{2}{*}{30.712} & \multirow{2}{*}{ Th } \\
\hline & right & & \\
\hline
\end{tabular}


Table 3: Dopamine, 3,4-Dihydroxyphenylacetic acid (DOPAC), homovanillic acid (HVA), and serotonin (5-HT) striatal content (nmol/mg) determined by HPLC.

\begin{tabular}{|c|c|c|}
\hline $\begin{array}{c}\text { Neurotransmitter } \\
\text { or } \\
\text { Metabolite }\end{array}$ & \multicolumn{2}{|c|}{$\begin{array}{c}\text { nmol/mg } \\
\text { (mean } \pm \text { SD) }\end{array}$} \\
\hline DA & Control & VMAT2 KD \\
\hline STR-L & $244 \pm 109$ & $351 \pm 168$ \\
\hline STR-R & $308 \pm 166$ & $100 \pm 97$ \\
\hline DOPAC & Control & VMAT2 KD \\
\hline STR-L & $34 \pm 7$ & $34 \pm 11$ \\
\hline STR-R & $38 \pm 4$ & $27 \pm 5$ \\
\hline HVA & Control & VMAT2 KD \\
\hline STR-L & $24 \pm 5$ & $25 \pm 5$ \\
\hline STR-R & $26 \pm 6$ & $19 \pm 4$ \\
\hline 5-HT & Control & VMAT2 KD \\
\hline STR-L & $17 \pm 2$ & $19 \pm 8$ \\
\hline STR-R & $19 \pm 6$ & $23 \pm 9$ \\
\hline
\end{tabular}




\section{Supplementary Tables}

Supplementary Table 1: $P$ values of the functional connectivity changes in regions of the DMN for rats with mild VMAT2 knockdown. Data were analyzed using paired t-tests.

\begin{tabular}{|c|c|c|c|c|c|c|c|c|c|c|c|c|}
\hline Mild & Hipp-R & Hipp-L & RSC-R & RSC-L & PaC-R & PaC-L & OFC-R & OFC-L & mPFC-R & mPFC-L & CgC-R \\
\hline CgC-L & 0.40 & 0.56 & 0.95 & 0.33 & 0.76 & 0.84 & 0.22 & 0.27 & 0.07 & 0.07 & 0.61 \\
\hline CgC-R & 0.30 & 0.41 & 0.83 & 0.29 & 0.84 & 0.86 & 0.17 & 0.23 & 0.03 & 0.08 & \\
\hline mPFC-L & 0.21 & 0.34 & 0.77 & 0.73 & 0.36 & 0.55 & 0.46 & 0.02 & 0.53 & & \\
\hline mPFC-R & 0.12 & 0.23 & 0.54 & 0.99 & 0.24 & 0.46 & 0.24 & 0.61 & & & \\
\hline OFC-L & 0.49 & 0.55 & 0.20 & 0.05 & 0.51 & 0.67 & 0.18 & & & & \\
\hline OFC-R & 0.31 & 0.20 & 0.26 & 0.13 & 0.23 & 0.45 & & & & & \\
\hline PaC-L & 0.61 & 0.39 & 0.84 & 0.08 & 0.31 & & & & & & \\
\hline PaC-R & 0.74 & 0.59 & 0.83 & 0.11 & & & & & & & \\
\hline RSC-L & 0.18 & 0.22 & 0.31 & & & & & & & & \\
\hline RSC-R & 0.75 & 0.65 & & & & & & & & & \\
\hline Hipp-L & 0.67 & & & & & & & & & & \\
\hline
\end{tabular}

1299

Supplementary Table 2: $P$ values of the functional connectivity changes in regions of the DMN for rats with moderate VMAT2 knockdown. Data were analyzed using paired t-tests.

\begin{tabular}{|c|c|c|c|c|c|c|c|c|c|c|c|}
\hline Moderate & Hipp-R & Hipp-L & RSC-R & RSC-L & PaC-R & PaC-L & OFC-R & OFC-L & mPFC-R & mPFC-L & CgC-R \\
\hline CgC-L & 0.05 & 0.02 & 0.07 & 0.11 & 0.24 & 0.22 & 0.05 & 0.03 & 0.03 & 0.04 & 0.07 \\
\hline CgC-R & 0.03 & 0.02 & 0.08 & 0.14 & 0.21 & 0.25 & 0.04 & 0.01 & 0.03 & 0.05 & \\
\hline mPFC-L & 0.01 & 0.03 & 0.03 & 0.09 & 0.05 & 0.04 & 0.04 & 0.04 & 0.06 & & \\
\hline mPFC-R & $\mathbf{0 . 0 0 5}$ & $\mathbf{0 . 0 0 7}$ & 0.01 & 0.03 & 0.03 & 0.03 & 0.06 & 0.01 & & & \\
\hline OFC-L & 0.08 & 0.10 & 0.04 & 0.13 & 0.24 & 0.21 & 0.05 & & & & \\
\hline OFC-R & 0.16 & 0.11 & 0.03 & 0.07 & 0.29 & 0.47 & & & & & \\
\hline PaC-L & 0.35 & 0.60 & 0.03 & 0.10 & 0.13 & & & & & & \\
\hline PaC-R & 0.42 & 0.32 & 0.06 & 0.22 & & & & & & & \\
\hline RSC-L & 0.10 & 0.07 & 0.02 & & & & & & & & \\
\hline RSC-R & 0.03 & 0.04 & & & & & & & & & \\
\hline Hipp-L & 0.15 & & & & & & & & & & \\
\hline
\end{tabular}


Supplementary Table 4: $P$ values of the functional connectivity changes in regions of the SMN for rats with moderate VMAT2 knockdown. Data were analyzed using paired t-tests.

\begin{tabular}{|c|c|c|c|c|c|c|c|}
\hline Moderate & Th-R & Th-L & SC-R & SC-L & MC-R & MC-L & STR-R \\
\hline STR-L & $\mathbf{0 . 0 1}$ & $\mathbf{0 . 0 0 4}$ & 0.02 & 0.05 & 0.03 & 0.04 & $\mathbf{0 . 0 0 7}$ \\
\hline STR-R & 0.02 & $\mathbf{0 . 0 0 8}$ & 0.06 & 0.04 & 0.16 & 0.07 & \\
\hline MC-L & 0.04 & 0.01 & 0.10 & 0.08 & 0.04 & & \\
\hline MC-R & 0.02 & 0.02 & 0.07 & & & & \\
\hline SC-L & $\mathbf{0 . 0 0 3}$ & $\mathbf{0 . 0 0 1}$ & 0.11 & & & & \\
\hline SC-R & 0.02 & $\mathbf{0 . 0 0 8}$ & & & & & \\
\hline Th-L & 0.07 & & & & & & \\
\hline Th-R & & & & & & & \\
\hline
\end{tabular}
Supplementary Table 5: $P$ values of the functional connectivity internetwork changes in regions of
the DMN and SMN for rats with mild VMAT2 knockdown. Data were analyzed using paired t-tests. Supplementary Table 5: $P$ values of the functional connectivity internetwork changes in regions of
the DMN and SMN for rats with mild VMAT2 knockdown. Data were analyzed using paired t-tests.

\begin{tabular}{|c|c|c|c|c|c|c|c|c|c|c|c|c|}
\hline Mild & Hipp-R & Hipp-L & RSC-R & RSC-L & PaC-R & PaC-L & OFC-R & OFC-L & mPFC-R & mPFC-L & CgC-R & CgC-L \\
\hline STR-L & 0.23 & 0.18 & 0.82 & 0.26 & 0.94 & 0.93 & $\mathbf{0 . 0 0 6}$ & $\mathbf{0 . 0 0 5}$ & 0.10 & 0.15 & 0.27 & 0.29 \\
\hline STR-R & 0.16 & 0.44 & 0.65 & 0.32 & 0.65 & 0.98 & $\mathbf{0 . 0 0 0 4}$ & 0.03 & 0.06 & 0.19 & 0.24 & 0.31 \\
\hline MC-L & 0.34 & 0.39 & 0.92 & 0.29 & 0.93 & 0.94 & 0.11 & 0.40 & 0.02 & 0.04 & 0.10 & 0.07 \\
\hline MC-R & 0.26 & 0.60 & 0.18 & 0.04 & 0.49 & 0.88 & 0.37 & 0.16 & 0.02 & 0.05 & 0.18 & 0.35 \\
\hline SC-L & 0.12 & 0.26 & 0.78 & 0.05 & 0.87 & 0.46 & $\mathbf{0 . 0 0 2}$ & 0.02 & 0.03 & 0.05 & 0.13 & 0.17 \\
\hline SC-R & 0.13 & 0.57 & 0.19 & $\mathbf{0 . 0 0 6}$ & 0.89 & 0.04 & 0.01 & 0.04 & 0.02 & 0.06 & 0.18 & 0.28 \\
\hline Th-L & 0.35 & 0.27 & 0.55 & 0.39 & 1.00 & 0.89 & 0.09 & 0.21 & 0.11 & 0.17 & 0.14 & 0.17 \\
\hline Th-R & 0.38 & 0.98 & 0.66 & 0.26 & 0.94 & 0.70 & 0.09 & 0.15 & 0.09 & 0.11 & 0.28 & 0.33 \\
\hline
\end{tabular}

Supplementary Table 3: $P$ values of the functional connectivity changes in regions of the SMN for rats with mild VMAT2 knockdown. Data were analyzed using paired t-tests.

\begin{tabular}{|c|c|c|c|c|c|c|c|}
\hline Mild & Th-R & Th-L & SC-R & SC-L & MC-R & MC-L & STR-R \\
\hline STR-L & 0.13 & 0.06 & 0.21 & 0.14 & 0.23 & 0.21 & 0.39 \\
\hline STR-R & 0.13 & 0.09 & 0.19 & 0.13 & 0.39 & 0.29 & \\
\hline MC-L & 0.24 & 0.16 & 0.47 & 0.16 & 0.46 & & \\
\hline MC-R & 0.29 & 0.23 & 0.38 & 0.21 & & & \\
\hline SC-L & 0.14 & 0.04 & 0.76 & & & & \\
\hline SC-R & 0.30 & 0.12 & & & & & \\
\hline Th-L & 0.79 & & & & & & \\
\hline Th-R & & & & & & & \\
\hline
\end{tabular}


Supplementary Table 6: $\boldsymbol{P}$ values of the functional connectivity internetwork changes in regions of the DMN and SMN for rats with moderate VMAT2 knockdown. Data were analyzed using paired ttests.

\begin{tabular}{|c|c|c|c|c|c|c|c|c|c|c|c|c|}
\hline Moderate & Hipp-R & Hipp-L & RSC-R & RSC-L & PaC-R & PaC-L & OFC-R & OFC-L & mPFC-R & mPFC-L & CgC-R & CgC-L \\
\hline STR-L & 0.02 & 0.02 & 0.02 & 0.11 & 0.16 & 0.30 & 0.03 & $\mathbf{0 . 0 0 3}$ & $\mathbf{0 . 0 0 6}$ & 0.01 & 0.07 & 0.09 \\
\hline STR-R & 0.08 & 0.08 & 0.04 & 0.14 & 0.30 & 0.63 & $\mathbf{0 . 0 0 8}$ & 0.01 & $\mathbf{0 . 0 0 2}$ & $\mathbf{0 . 0 0 3}$ & 0.04 & 0.07 \\
\hline MC-L & 0.03 & 0.03 & $\mathbf{0 . 0 0 3}$ & 0.03 & 0.45 & 0.46 & 0.04 & 0.07 & $\mathbf{0 . 0 0 3}$ & $\mathbf{0 . 0 0 3}$ & 0.02 & 0.02 \\
\hline MC-R & 0.03 & 0.05 & 0.05 & 0.18 & 0.52 & 0.97 & 0.19 & $\mathbf{0 . 0 0 8}$ & $\mathbf{0 . 0 1}$ & $\mathbf{0 . 0 0 7}$ & 0.03 & 0.03 \\
\hline SC-L & $\mathbf{0 . 0 0 4}$ & $\mathbf{0 . 0 0 8}$ & $\mathbf{0 . 0 0 8}$ & 0.08 & 0.11 & 0.44 & 0.05 & 0.02 & $\mathbf{0 . 0 0 3}$ & $\mathbf{0 . 0 0 4}$ & 0.04 & 0.06 \\
\hline SC-R & 0.02 & 0.08 & 0.06 & 0.35 & 0.27 & 0.94 & 0.07 & 0.04 & $\mathbf{0 . 0 0 1}$ & $\mathbf{0 . 0 0 3}$ & 0.04 & 0.07 \\
\hline Th-L & 0.06 & 0.02 & 0.09 & 0.10 & 0.17 & 0.15 & 0.06 & 0.03 & 0.02 & 0.03 & 0.03 & 0.04 \\
\hline Th-R & 0.03 & 0.10 & 0.09 & 0.23 & 0.15 & 0.13 & 0.14 & 0.08 & 0.02 & 0.04 & 0.07 & 0.15 \\
\hline
\end{tabular}

Supplementary Table 7: Bonferroni-Sidak corrected $P$ values for graph theoretical measures of the global mean connection distance in mild and moderate VMAT2 knockdown rats. Data were analyzed using paired t-tests.

\begin{tabular}{|c|c|c|}
\hline Region & Mild & Moderate \\
\hline CgC-L & 0.39 & 0.48 \\
\hline CgC-R & 0.37 & 0.45 \\
\hline mPFC-L & 0.41 & 0.24 \\
\hline mPFC-R & 0.28 & 0.10 \\
\hline OFC-L & 0.87 & 0.81 \\
\hline OFC-R & 0.67 & 0.89 \\
\hline PaC-L & 0.68 & 0.96 \\
\hline PaC-R & 0.66 & 0.83 \\
\hline RSC-L & 0.18 & 0.96 \\
\hline RSC-R & 0.97 & 0.38 \\
\hline Hipp-L & 0.50 & 0.42 \\
\hline Hipp-R & 0.34 & 0.35 \\
\hline STR-L & 0.15 & $\mathbf{0 . 0 1}$ \\
\hline STR-R & 0.18 & 0.09 \\
\hline MC-L & 0.27 & 0.64 \\
\hline MC-R & 0.29 & 0.48 \\
\hline SC-L & 0.16 & 0.08 \\
\hline SC-R & 0.29 & 0.35 \\
\hline Th-L & 0.12 & $\mathbf{0 . 0 3}$ \\
\hline Th-R & 0.28 & 0.09 \\
\hline
\end{tabular}


Supplementary Table 8: Primers used to amplify the SIc18a2 and the lacZ loci in the Surveyor assay and DNA expected fragments sizes.

\begin{tabular}{|c|c|c|c|}
\hline SgRnAs & $\begin{array}{c}\text { Forward Primer } \\
\mathbf{5}^{\prime} \mathbf{-} \mathbf{3}^{\prime}\end{array}$ & $\begin{array}{c}\text { Reverse Primer } \\
\mathbf{5}^{\prime} \mathbf{-} \mathbf{3}^{\prime}\end{array}$ & Fragment size (bp) \\
\hline S/c18a2 & CTTGGGGATCCTCTAAGGCAG & TACAGCGCGGTTCTTCAACT & 313_280 \\
\hline lacZ & GTCGTGACTGGGAAAACCCT & TTGTTCCCACGGAGAATCCG & 200_80 \\
\hline
\end{tabular}

1354

Supplementary Table 9: $\left[{ }^{11} \mathrm{C}\right] \mathrm{DTBZ},\left[{ }^{11} \mathrm{C}\right]$ methylphenidate, $\left[{ }^{11} \mathrm{C}\right]$ raclopride and $\left[{ }^{18} \mathrm{~F}\right] \mathrm{GE}-180$ injected and molar activities.

\begin{tabular}{|c|c|c|}
\hline Radioligand & $\begin{array}{l}\text { Injected Activity (Mean } \pm \text { SD) } \\
\text { MBq }\end{array}$ & 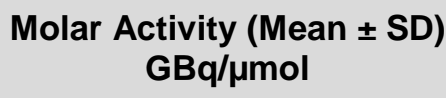 \\
\hline$\left[{ }^{11} \mathrm{C}\right] \mathrm{DTBZ}$ & $25 \pm 1$ & $93 \pm 35$ \\
\hline$\left[{ }^{11} \mathrm{C}\right] \mathrm{MP}$ & $25 \pm 1$ & $57 \pm 14$ \\
\hline$\left[{ }^{11} \mathrm{C}\right] \mathrm{RAC}$ & $25 \pm 1$ & $88 \pm 41$ \\
\hline$\left[{ }^{18} \mathrm{~F}\right] \mathrm{GE}-180$ & $24 \pm 3$ & $576 \pm 283$ \\
\hline
\end{tabular}

Supplementary Table 10: $\left[{ }^{11} \mathrm{C}\right]$ raclopride, $\left[{ }^{11} \mathrm{C}\right] \mathrm{DTBZ},\left[{ }^{11} \mathrm{C}\right]$ flumazenil injected and molar activities.

\begin{tabular}{|c|c|c|}
\hline Radioligand & $\begin{array}{l}\text { Injected Activity (Mean } \pm \text { SD) } \\
\text { MBq }\end{array}$ & $\begin{array}{c}\text { Molar Activity (Mean } \pm \text { SD) } \\
\text { GBq/ } / \mu \mathrm{mol}\end{array}$ \\
\hline$\left[{ }^{11} \mathrm{C}\right] \mathrm{RAC}$ t0 & $27 \pm 4$ & $108 \pm 49$ \\
\hline$\left[{ }^{11} \mathrm{C}\right] \mathrm{RAC}$ t8 & $28 \pm 1$ & $115 \pm 41$ \\
\hline$\left[{ }^{11} \mathrm{C}\right] \mathrm{DTBZ}$ & $27 \pm 1$ & $180 \pm 48$ \\
\hline$\left[{ }^{11} \mathrm{C}\right] \mathrm{FMZ}$ & $27 \pm 1$ & $109 \pm 40$ \\
\hline
\end{tabular}

